\title{
Vladimír Horák
}

Les complexes linéaires tangents des congruences de droites

Czechoslovak Mathematical Journal, Vol. 13 (1963), No. 2, 166-188

Persistent URL: http://dml.cz/dmlcz/100561

\section{Terms of use:}

(C) Institute of Mathematics AS CR, 1963

Institute of Mathematics of the Czech Academy of Sciences provides access to digitized documents strictly for personal use. Each copy of any part of this document must contain these Terms of use.

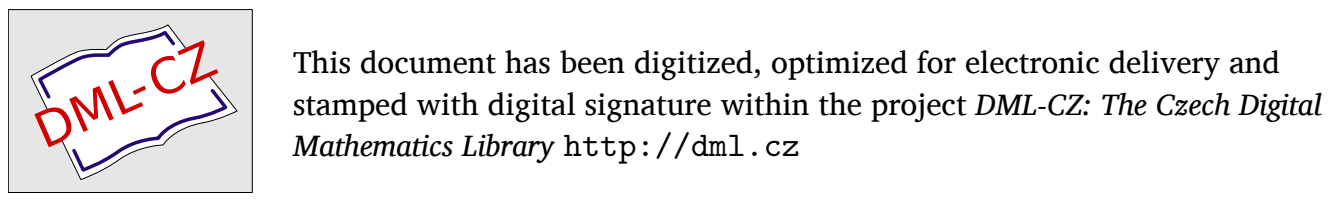




\title{
LES COMPLEXES LINÉAIRES TANGENTS DES CONGRUENCES DE DROITES
}

\author{
Vladimír Horák, Brno \\ (Reçu le 21 janvier 1961)
}

\begin{abstract}
L'auteur décrit la structure géométrique du voisinage différentiel du premier ordre des droites des congruences non paraboliques et étudie la variété formée par les images secondaires de Klein des complexes tangents de ces congruences.
\end{abstract}

1. Au Mémoire [1] on considère une congruence non-parabolique $L$ de droites dans l'espace projectif $P_{3}$, en faisant usage du repère

$$
A_{1}, A_{2}, A_{3}, A_{4}
$$

assujetti à la condition

$$
\left[A_{1} A_{2} A_{3} A_{4}\right]=1,
$$

et tel que les transformations infinitésimales sont données par les relations

$$
\mathrm{d} A_{i}=\omega_{i 1} A_{1}+\omega_{i 2} A_{2}+\omega_{i 3} A_{3}+\omega_{i 4} A_{4}, \quad i=1,2,3,4
$$

où

$$
\omega_{11}+\omega_{22}+\omega_{33}+\omega_{44}=0 .
$$

Soit le point $A_{1}\left(A_{2}\right)$ le premier (second) foyer, le plan $\left[A_{1} A_{2} A_{4}\right]\left(\left[A_{1} A_{2} A_{3}\right]\right)$ le premier (second) plan focal et les droites $\left[A_{1} A_{3}\right],\left[A_{2} A_{4}\right]$ soient les transformées de Laplace de la droite $\left[A_{1} A_{2}\right]$ de la congruence $L$. Alors la congruence (maintenant orientée) est déterminée par le système des équations différentielles

$$
\begin{aligned}
& \mathrm{d} A_{1}=\omega_{11} A_{1}+\alpha_{1} \omega_{2} A_{2}+\omega_{1} A_{3}, \\
& \mathrm{~d} A_{2}=\alpha_{2} \omega_{1} A_{1}+\omega_{22} A_{2}+\omega_{2} A_{4}, \\
& \mathrm{~d} A_{3}=\omega_{31} A_{1}+\omega_{32} A_{2}+\omega_{33} A_{3}+\beta_{2} \omega_{1} A_{4}, \\
& \mathrm{~d} A_{4}=\omega_{41} A_{1}+\omega_{42} A_{2}+\beta_{1} \omega_{2} A_{3}+\omega_{44} A_{4} ;
\end{aligned}
$$

la comparaison des relations de ci-dessus avec les relations (1.3) donne

$$
\begin{gathered}
\omega_{14}=\omega_{23}=0, \quad \omega_{12}=\alpha_{1} \omega_{2}, \omega_{21}=\alpha_{2} \omega_{1}, \omega_{34}=\beta_{2} \omega_{1}, \\
\omega_{43}=\beta_{1} \omega_{2},
\end{gathered}
$$


où nous avons introduit la notation

$$
\omega_{13}=\omega_{1}, \quad \omega_{24}=\omega_{2} .
$$

Les conditions d'intégrabilité du système (1.6) des équations de Pfaff sont

$$
\begin{aligned}
& {\left[\omega_{32} \omega_{1}\right]+\left[\left(\mathrm{d} \alpha_{1}+\alpha_{1}\left(2 \omega_{22}-\omega_{11}-\omega_{44}\right)\right) \omega_{2}\right]=0,} \\
& {\left[\left(\mathrm{~d} \alpha_{2}+\alpha_{2}\left(2 \omega_{11}-\omega_{22}-\omega_{33}\right)\right) \omega_{1}\right]+\left[\omega_{41} \omega_{2}\right]=0,} \\
& {\left[\omega_{41} \omega_{1}\right]-\left[\left(\mathrm{d} \beta_{1}+\beta_{1}\left(\omega_{22}+\omega_{33}-2 \omega_{44}\right)\right) \omega_{2}\right]=0,} \\
& {\left[\left(\mathrm{~d} \beta_{2}+\beta_{2}\left(\omega_{11}+\omega_{44}-2 \omega_{33}\right)\right) \omega_{1}\right]-\left[\omega_{32} \omega_{2}\right]=0 .}
\end{aligned}
$$

Finalement, on a encore

$$
\left[\mathrm{d} \omega_{1}\right]=\left[\left(\omega_{11}-\omega_{33}\right) \omega_{1}\right], \quad\left[\mathrm{d} \omega_{2}\right]=\left[\left(\omega_{22}-\omega_{44}\right) \omega_{2}\right] .
$$

En considérant le repère

$$
\begin{aligned}
E_{1}=\left[A_{2} A_{3} A_{4}\right], \quad E_{2} & =-\left[A_{1} A_{3} A_{4}\right], \quad E_{3}=\left[A_{1} A_{2} A_{4}\right], \\
E_{4} & =-\left[A_{1} A_{2} A_{3}\right],
\end{aligned}
$$

corrélatif au repère $(1.1)$, on obtient de (1.5) les relations

$$
\begin{aligned}
\mathrm{d} E_{1}+\omega_{11} E_{1}+\alpha_{2} \omega_{1} E_{2} & +\omega_{31} E_{3}+\omega_{41} E_{4}=0, \\
\mathrm{~d} E_{2}+\alpha_{1} \omega_{2} E_{1}+\omega_{22} E_{2} & +\omega_{32} E_{3}+\omega_{42} E_{4}=0, \\
\mathrm{~d} E_{3}+\omega_{1} E_{1} & +\omega_{33} E_{3}+\beta_{1} \omega_{2} E_{4}=0, \\
\mathrm{~d} E_{4}+\omega_{2} E_{2}+\beta_{2} \omega_{1} E_{3}+\omega_{44} E_{4}=0 &
\end{aligned}
$$

et pour le repère réglé $\left[A_{1} A_{2}\right],\left[A_{1} A_{3}\right],\left[A_{2} A_{4}\right],\left[A_{1} A_{4}\right],\left[A_{2} A_{3}\right],\left[A_{3} A_{4}\right]$ les relations

$$
\begin{aligned}
& \mathrm{d}\left[A_{1} A_{2}\right]=\left(\omega_{11}+\omega_{22}\right)\left[A_{1} A_{2}\right]+ \\
& +\omega_{2}\left[A_{1} A_{4}\right]-\omega_{1}\left[A_{2} A_{3}\right] \text {, } \\
& \mathrm{d}\left[A_{1} A_{3}\right]=\quad \omega_{32}\left[A_{1} A_{2}\right]+\left(\omega_{11}+\omega_{33}\right)\left[A_{1} A_{3}\right]+ \\
& +\beta_{2} \omega_{1}\left[A_{1} A_{4}\right]+\alpha_{1} \omega_{2}\left[A_{2} A_{3}\right] \text {, } \\
& \mathrm{d}\left[A_{2} A_{4}\right]=-\omega_{41}\left[A_{1} A_{2}\right]+\left(\omega_{22}+\omega_{44}\right)\left[A_{2} A_{4}\right]+ \\
& +\alpha_{2} \omega_{1}\left[A_{1} A_{4}\right]+\beta_{1} \omega_{2}\left[A_{2} A_{3}\right] \text {, } \\
& \mathrm{d}\left[A_{1} A_{4}\right]=\quad \omega_{42}\left[A_{1} A_{2}\right]+\beta_{1} \omega_{2}\left[A_{1} A_{3}\right]+\alpha_{1} \omega_{2}\left[A_{2} A_{4}\right]+ \\
& +\left(\omega_{11}+\omega_{44}\right)\left[A_{1} A_{4}\right] \quad+\omega_{1}\left[A_{3} A_{4}\right], \\
& \mathrm{d}\left[A_{2} A_{3}\right]=-\omega_{31}\left[A_{1} A_{2}\right]+\alpha_{2} \omega_{1}\left[A_{1} A_{3}\right]+\beta_{2} \omega_{1}\left[A_{2} A_{4}\right]+ \\
& +\left(\omega_{22}+\omega_{33}\right)\left[A_{2} A_{3}\right] \quad-\omega_{2}\left[A_{3} A_{4}\right] \text {, } \\
& \mathrm{d}\left[A_{3} A_{4}\right]=\quad-\omega_{41}\left[A_{1} A_{3}\right]+\omega_{32}\left[A_{2} A_{4}\right]+ \\
& +\omega_{31}\left[A_{1} A_{4}\right]-\omega_{42}\left[A_{2} A_{3}\right]+\left(\omega_{33}+\omega_{44}\right)\left[A_{3} A_{4}\right] \text {. }
\end{aligned}
$$

Signalons encore que l'on a

$$
\begin{aligned}
\mathrm{d}^{2}\left[A_{1} A_{2}\right]= & {\left[\mathrm{d}\left(\omega_{11}+\omega_{22}\right)+\left(\omega_{11}+\omega_{22}\right)^{2}+\omega_{31} \omega_{1}+\omega_{42} \omega_{2}\right]\left[A_{1} A_{2}\right]+} \\
& +\left(\beta_{1} \omega_{2}^{2}-\alpha_{2} \omega_{1}^{2}\right)\left[A_{1} A_{3}\right]+\left(\alpha_{1} \omega_{2}^{2}-\beta_{2} \omega_{1}^{2}\right)\left[A_{2} A_{4}\right]+ \\
& +\left[\mathrm{d} \omega_{2}+\left(2 \omega_{11}+\omega_{22}+\omega_{44}\right) \omega_{2}\right]\left[A_{1} A_{4}\right]- \\
& -\left[\mathrm{d} \omega_{1}+\left(\omega_{11}+2 \omega_{22}+\omega_{33}\right) \omega_{1}\right]\left[A_{2} A_{3}\right]+2 \omega_{1} \omega_{2}\left[A_{3} A_{4}\right]
\end{aligned}
$$




$$
\begin{aligned}
\mathrm{d}^{2}\left[E_{3} E_{4}\right]= & {\left[-\mathrm{d}\left(\omega_{33}+\omega_{44}\right)+\left(\omega_{33}+\omega_{44}\right)^{2}+\omega_{31} \omega_{1}+\omega_{42} \omega_{2}\right]\left[E_{3} E_{4}\right]-} \\
& -\left(\alpha_{1} \omega_{2}^{2}-\beta_{2} \omega_{1}^{2}\right)\left[E_{1} E_{3}\right]-\left(\beta_{1} \omega_{2}^{2}-\alpha_{2} \omega_{1}^{2}\right)\left[E_{2} E_{4}\right]+ \\
& +\left[\mathrm{d} \omega_{2}-\left(2 \omega_{33}+\omega_{44}+\omega_{22}\right) \omega_{2}\right]\left[E_{2} E_{3}\right]- \\
& -\left[\mathrm{d} \omega_{1}-\left(\omega_{33}+2 \omega_{44}+\omega_{11}\right) \omega_{1}\right]\left[E_{1} E_{4}\right]+2 \omega_{1} \omega_{2}\left[E_{1} E_{2}\right] .
\end{aligned}
$$

Les lignes asymptotiques sur la surface focale $\left(A_{1}\right)$, resp. $\left(A_{2}\right)$ sont déterminées par l'équation différentielle

$$
\beta_{2} \omega_{1}^{2}+\alpha_{1} \omega_{2}^{2}=0, \quad \text { resp. } \alpha_{2} \omega_{1}^{2}+\beta_{1} \omega_{2}^{2}=0 .
$$

On voit sans peine des relations précédentes qu'on obtient la dualisation $L^{*}$ de la congruence $L$ par les transformations

$$
\left(\begin{array}{rrrrrrrr}
A_{1} & A_{2} & A_{3} & A_{4} & E_{1} & E_{2} & E_{3} & E_{4} \\
E_{3} & -E_{4} & -E_{1} & E_{2} & -A_{3} & A_{4} & A_{1} & -A_{2}
\end{array}\right)
$$

$$
\left(\begin{array}{rrrrrrrrrrrrrr}
\omega_{1} & \omega_{2} & \alpha_{1} & \alpha_{2} & \beta_{1} & \beta_{2} & \omega_{11} & \omega_{22} & \omega_{33} & \omega_{44} & \omega_{31} & \omega_{42} & \dot{\omega}_{32} & \omega_{41} \\
\omega_{1} & \omega_{2} & \beta_{1} & \beta_{2} & \alpha_{1} & \alpha_{2} & -\omega_{33} & -\omega_{44} & -\omega_{11} & -\omega_{22} & \omega_{31} & \omega_{42} & -\omega_{41} & -\omega_{32}
\end{array}\right) .
$$

D'après la classification de E. ČECH nous distinguons les dix types suivants des congruences nonparaboliques ([1], p. 264-265):

Type I: $\alpha_{1} \alpha_{2} \beta_{1} \beta_{2} \neq 0-$ des congruences dont les surfaces focales $\left(A_{1}\right)$ et $\left(A_{2}\right)$ sont non-développables.

Type II: $\alpha_{1} \beta_{1} \beta_{2} \neq 0=\alpha_{2}$, resp. $\alpha_{2} \beta_{1} \beta_{2} \neq 0=\alpha_{1}$ - la surface focale $\left(A_{1}\right)$, resp. $\left(A_{2}\right)$ est non-développable et la surface focale $\left(A_{2}\right)$, resp. $\left(A_{1}\right)$ dégénère en une courbe non-rectiligne.

Type II*: $\alpha_{1} \alpha_{2} \beta_{1} \neq 0=\beta_{2}$, resp. $\alpha_{1} \alpha_{2} \beta_{2} \neq 0=\beta_{1}$ - corrélatif au type II.

Type III: $\alpha_{1} \beta_{2} \neq 0=\alpha_{2}=\beta_{1}$, resp. $\alpha_{2} \beta_{1} \neq 0=\alpha_{1}=\beta_{2}$ - la surface focale $\left(A_{1}\right)$, resp. $\left(A_{2}\right)$ est non-développable et $\left(A_{2}\right)$, resp. $\left(A_{1}\right)$ est une droite. $\left.{ }^{1}\right)$

Type IV: $\alpha_{2} \beta_{2} \neq 0=\alpha_{1}=\beta_{1}$, resp. $\alpha_{1} \beta_{1} \neq 0=\alpha_{2}=\beta_{2}$ - la surface focale $\left(A_{1}\right)$, resp. $\left(A_{2}\right)$ est une courbe et $\left(A_{1}\right)$, resp. $\left(A_{2}\right)$ est une surface développable.

Type V: $\beta_{1} \beta_{2} \neq 0=\alpha_{1}=\alpha_{2}-\left(A_{1}\right)$ et $\left(A_{2}\right)$ sont des courbes.

Type $\mathrm{V}^{*}: \alpha_{1} \alpha_{2} \neq 0=\beta_{1}=\beta_{2}-$ corrélatif au type $\mathrm{V}$.

Type VI: $\beta_{2} \neq 0=\alpha_{1}=\alpha_{2}=\beta_{1}$, resp. $\beta_{1} \neq 0=\alpha_{1}=\alpha_{2}=\beta_{2}-\left(A_{1}\right)$, resp. $\left(A_{2}\right)$ est une courbe directrice non rectiligne et $\left(A_{2}\right)$, resp. $\left(A_{1}\right)$ est une droite. $\left.{ }^{1}\right)$

Type VI*: $\alpha_{2} \neq 0=\alpha_{1}=\beta_{1}=\beta_{2}$, resp. $\alpha_{1} \neq 0=\alpha_{2}=\beta_{1}=\beta_{2}$ corrélatif au type VI.

Type VII: $\alpha_{1}=\alpha_{2}=\beta_{1}=\beta_{2}=0-\left(A_{1}\right)$ et $\left(A_{2}\right)$ sont des droites et Lest une congruence linéaire nonparabolique.

${ }^{1}$ ) Les relations $\alpha_{1}=\beta_{2}=0$, resp. $\alpha_{2}=\beta_{1}=0$ entraînent $\omega_{32}=0$, resp. $\omega_{41}=0$. 
2. Le complexe linéaire

$$
\begin{aligned}
\Omega \equiv a_{1}\left[A_{1} A_{2}\right]+a_{2}\left[A_{1} A_{3}\right] & +a_{3}\left[A_{2} A_{4}\right]+a_{4}\left[A_{1} A_{4}\right]+a_{5}\left[A_{2} A_{3}\right]+ \\
& +a_{6}\left[A_{3} A_{4}\right]
\end{aligned}
$$

est appellé complexe tangent de la congruence $L$ le long de la droite $\left[A_{1} A_{2}\right]$ si les relations

$$
\Omega \cdot\left[A_{1} A_{2}\right]=\Omega \cdot \mathrm{d}\left[A_{1} A_{2}\right]=0
$$

sont vérifiées.

En vertu de ces relations on obtient le complexe mentionné sous la forme

$$
\Omega \equiv a_{1}\left[A_{1} A_{2}\right]+a_{2}\left[A_{1} A_{3}\right]+a_{3}\left[A_{2} A_{4}\right] .
$$

En général, il y a $\infty^{2}$ des complexes tangents d'une congruence Larbitraire le long de la génératrice $\left[A_{1} A_{2}\right]$. Les images secondaires de ces complexes remplissent dans l'espace à cinq dimensions de Klein le plan

$$
\bar{P}_{2} \equiv\left\{\left[A_{1} A_{2}\right],\left[A_{1} A_{3}\right],\left[A_{2} A_{4}\right]\right\}
$$

qui coupe l'hyperquadrique de Klein (K-quadrique) aux K-droites $\overline{\left[A_{1} A_{2}\right]\left[A_{1} A_{3}\right]}$ et $\overline{\left[A_{1} A_{2}\right]\left[A_{2} A_{4}\right]}$ qui possèdent le K-point $\left[A_{1} A_{2}\right]$ commun et alors le plan (2.4) est tangent à la $K$-quadrique au K-point $\left[A_{1} A_{2}\right]$. Dans la polarité par rapport à la $K$-quadrique le plan (2.4) est associé au plan

$$
\bar{P}_{2} \equiv\left\{\left[A_{1} A_{2}\right],\left[A_{1} A_{4}\right],\left[A_{2} A_{3}\right]\right\} ;
$$

ce dernier est tangent au K-point $\left[A_{1} A_{2}\right]$ à la $K$-quadrique et en même temps tangent au même K-point à la surface $(\Psi) \equiv\left(\left[A_{1} A_{2}\right)\right]$ qui est l'image de Klein de la congruence L sur la K-quadrique.

Le complexe tangent $\Omega$ est un complexe spécial si et seulement si l'on a

$$
a_{2} a_{3}=0,
$$

de sorte que les images secondaires des complexes tangents spéciaux sont situées sur les $K$-droites déjà mentionnées qui sont l'intersection du plan (2.4) avec la K-quadrique.

En général, aucun des complexes tangents (2.3) ne contient le voisinage du second ordre de la droite $\left[A_{1} A_{2}\right]$ de $L$, c.-à-d. il n'est pas un complexe osculateur. L'auteur a démontré dans [3] que le complexe osculateur existe uniquement dans le cas des congruences $W\left(\alpha_{1} \alpha_{2} \beta_{1} \beta_{2} \neq 0\right)$ et des congruences du type IV. Mais chacun des complexes tangents de la droite $\left[A_{1} A_{2}\right]$ contient le voisinage du $2^{\mathrm{e}}$ ordre de certaines surfaces réglées de la congruence $L$ ce qui est le sujet de nos considérations suivantes.

La condition nécessaire et suffisante pour que le complexe $\Omega$ contienne le voisinage du second ordre de la droite $\left[A_{1} A_{2}\right]$, ou au moins le voisinage du second ordre d'une 
surface reglée qui passe par la droite $\left[A_{1} A_{2}\right]$ de la congruence $L$, est que dans tout le voisinage ou le long d'une surface réglée, la relation

$$
\Omega \cdot \mathrm{d}^{2}\left[A_{1} A_{2}\right]=0
$$

soit satisfaite.

En substituant dans cette relation pour $\Omega$ et $\mathrm{d}^{2}\left[A_{1} A_{2}\right]$ d'après (2.3) et (1.13) on obtient

$$
2 a_{1} \omega_{1} \omega_{2}-a_{2}\left(\alpha_{1} \omega_{2}^{2}-\beta_{2} \omega_{1}^{2}\right)-a_{3}\left(\beta_{1} \omega_{2}^{2}-\alpha_{2} \omega_{1}^{2}\right)=0
$$

cette équation est linéaire en $a_{1}, a_{2}, a_{3}$ et quadratique en $\omega_{1}: \omega_{2}$.

Supposons tout d'abord que Lest une congruence du type I, alors

$$
\alpha_{1} \alpha_{2} \beta_{1} \beta_{2} \neq 0 \text {. }
$$

(Les autres types voir chap. 4.)

Soit donné le rapport $a_{1}: a_{2}: a_{3}\left(a_{2}\right.$ et $a_{3}$ ne sont pas en même temps nuls) ensuite la relation (2.8) détermine deux couches de surfaces dans la congruence $L$; le complexe $\Omega\left(a_{1}: a_{2}: a_{3}\right)$ contient le voisinage du $2^{\mathrm{e}}$ ordre de deux surfaces qui passent par la droite $\left[A_{1} A_{2}\right]$ et chacune de ces surfaces appartient à une des couches mentionnées. On obtient les équations différentielles de ces couches comme racines de l'équation quadratique (2.8), c.-à̀-d.

$$
\begin{gathered}
\left(\frac{\omega_{1}}{\omega_{2}}\right)_{1,2}=\frac{-a_{1} \pm \sqrt{ }\left[a_{1}^{2}+\left(\alpha_{1} a_{2}+\beta_{1} a_{3}\right)\left(\beta_{2} a_{2}+\alpha_{2} a_{3}\right)\right]}{\beta_{2} a_{2}+\alpha_{2} a_{3}} \\
\left(=\frac{-\left(\alpha_{1} a_{2}+\beta_{1} a_{3}\right)}{-a_{1} \mp \sqrt{ }\left[a_{1}^{2}+\left(\alpha_{1} a_{2}+\beta_{1} a_{3}\right)\left(\beta_{2} a_{2}+\alpha_{2} a_{3}\right)\right]}\right) .
\end{gathered}
$$

Si les relations $a_{2}=a_{3}=0 \neq a_{1}$ sont vraies, alors au lieu de (2.8) on obtient $\omega_{1} \omega_{2}=0$, c.-à-d. les couches des surfaces développables.

Les complexes pour lesquels les deux surfaces mentionnées sont confondues, sont déterminées par la relation quadratique en $a_{1}, a_{2}, a_{3}$ :

$$
a_{1}^{2}+\alpha_{1} \beta_{2} a_{2}^{2}+\left(\alpha_{1} \alpha_{2}+\beta_{1} \beta_{2}\right) a_{2} a_{3}+\beta_{1} \alpha_{2} a_{3}^{2}=0 ;
$$

alors l'image de ces complexes dans le plan (2.4) est la conique dont l'équation dans les coordonées locales par rapport au triangle fondamental $\left[A_{1} A_{2}\right],\left[A_{1} A_{3}\right],\left[A_{2} A_{4}\right]$ est (2.11). Dorénavant, nous voulons désigner cette conique par $F$.

Le discriminant de la conique (2.11) est déterminé par la relation

$$
D \equiv\left|\begin{array}{ccc}
1, & 0, & 0 \\
0, & \alpha_{1} \beta_{2}, & \frac{1}{2}\left(\alpha_{1} \alpha_{2}+\beta_{1} \beta_{2}\right) \\
0, \frac{1}{2}\left(\alpha_{1} \alpha_{2}+\beta_{1} \beta_{2}\right), & \beta_{1} \alpha_{2}
\end{array}\right|=\frac{1}{4}\left(\alpha_{1} \alpha_{2}-\beta_{1} \beta_{2}\right)^{2} ;
$$


en général la conique $F$ n'est pas singulière. Si $F$ est singulière, alors son point singulier est donné par la relation

$$
a_{1}=0, \quad a_{2}: a_{3}=-\beta_{1}: \alpha_{1} \quad\left(=-\alpha_{2}: \beta_{2}\right) .
$$

On a alors le théorème:

La condition nécessaire et suffisante pour que la congruence $L d u$ type I soit une congruence $W$ est: la conique $F$ déterminée par l'équation (2.11), où $\alpha_{1} \alpha_{2} \beta_{1} \beta_{2} \neq 0$, est singulière. Le point singulier (2.13) de cette conique est l'image secondaire du complexe osculateur de la droite correspondante de la congruence $W$.

Notons encore: La condition nécessaire et suffisante pour que la conique $F$ soit composée de deux droites confondues est: tous les mineurs du $2^{\mathrm{e}}$ ordre du déterminant (2.12) sont nuls, c.-à-d. on a $\alpha_{1} \alpha_{2}=\beta_{1} \beta_{2}=\alpha_{1} \beta_{2}=\beta_{1} \alpha_{2}=0$; mais ces relations sont en contradiction avec notre supposition (2.9). Alors, si L est une congruence du type I, la conique $F$ ne peut pas être dégénerée de la manière énoncée.

Les points d'intersection de la droite $a_{1}=0$ avec la conique $(2.11)$ sont $\left(0,-\beta_{1}\right.$, $\left.\alpha_{1}\right),\left(0,-\alpha_{2}, \beta_{2}\right)$ et ils ne sont pas confondues tant que $\alpha_{1} \alpha_{2}-\beta_{1} \beta_{2} \neq 0$. Le rapport anharmonique $I$ de ces points et des sommets $(0,1,0),(0,0,1)$ du triangle fondamental dans le plan (2.4) est donné par la relation $\left(\alpha_{1} \alpha_{2}-\beta_{1} \beta_{2} \neq 0\right)$

$$
I=\frac{\alpha_{1} \alpha_{2}}{\beta_{1} \beta_{2}}
$$

ce qui est l'invariant classique de Wälsch (voir. p. ex. [1], p. 264). La signification géométrique des points $\left(0,-\beta_{1}, \alpha_{1}\right)$ et $\left(0,-\alpha_{2}, \beta_{2}\right)$ voir $\mathrm{p} .175$.

On voit sans peine que le $K$-point $\left[A_{1} A_{2}\right]$ (c.-à-d. $\left.(1,0,0)\right)$ et la droite $\left[A_{1} A_{3}\right]$. - $\left[A_{2} A_{4}\right]\left(a_{1}=0\right)$ sont le pôle et la polaire de la conique (2.11).

Soit

$$
\left.\omega_{2}=c \omega_{1}, \quad c \neq 0^{2}\right)
$$

l'équation différentielle d'une couche de surfaces réglées dans $L$. Nous allons déterminer les complexes qui possèdent le voisinage du $2^{\mathrm{e}}$ ordre de ces surîaces. En substituant d'après (2.15) dans (2.8) on obtient

$$
2 c a_{1}+\left(\beta_{2}-\alpha_{1} c^{2}\right) a_{2}+\left(\alpha_{2}-\beta_{1} c^{2}\right) a_{3}=0 .
$$

On a alors: Les images secondaires des complexes tangents qui possèdent le voisinage du $2^{e}$ ordre des surfaces (2.15) remplissent dans le plan (2.4) une droite; dans l'espace $P_{3}$ ces complexes forment un faisceau.

En posant d'après (2.16)

$$
a_{1} c+\left(\beta_{2}-\alpha_{1} c^{2}\right) a_{2}=-a_{1} c+\left(\beta_{1} c^{2}-\alpha_{2}\right) a_{3}=\lambda, \quad a_{1}=\mu
$$

\footnotetext{
2) Par la relation (2.15) on a exclu les couches des surfaces développables. Pour ces couches on peut facilement obtenir les résultats relatifs.
} 
on obtient l'équation du faisceau mentionné sous la forme $(\lambda, \mu$ sont des paramètres variables, $\left.\beta_{1}-\alpha_{1} c^{2} \neq 0, \alpha_{2}-\beta_{1} c^{2} \neq 0\right)$

$$
\begin{aligned}
\Omega= & \sigma\left\{\mu\left(\left[A_{1} A_{2}\right]-\frac{c}{\beta_{2}-\alpha_{1} c^{2}}\left[A_{1} A_{3}\right]-\frac{c}{\alpha_{2}-\beta_{1} c^{2}}\left[A_{2} A_{4}\right]\right)+\right. \\
& \left.+\lambda\left(\frac{1}{\beta_{2}-\alpha_{1} c^{2}}\left[A_{1} A_{3}\right]-\frac{1}{\alpha_{2}-\beta_{1} c^{2}}\left[A_{2} A_{4}\right]\right)\right\}= \\
= & \bar{\sigma}\left\{\mu \left(\left(\alpha_{2}-\beta_{1} c^{2}\right)\left(\beta_{2}-\alpha_{1} c^{2}\right)\left[A_{1} A_{2}\right]-c\left(\alpha_{2}-\beta_{1} c^{2}\right)\left[A_{1} A_{3}\right]-\right.\right. \\
& \left.-c\left(\beta_{2}-\alpha_{1} c^{2}\right)\left[A_{2} A_{4}\right]\right)+\lambda\left(\left(\alpha_{2}-\beta_{1} c^{2}\right)\left[A_{1} A_{3}\right]-\right. \\
& \left.\left.-\left(\beta_{2}-\alpha_{1} c^{2}\right)\left[A_{2} A_{4}\right]\right)\right\}, \quad \sigma \bar{\sigma}, \neq 0 ;
\end{aligned}
$$

les complexes spéciaux dans ce faisceau résultent pour $\lambda= \pm c \mu$. Chacun des complexes de ce faisceau contient encore le voisinage du $2^{\mathrm{e}}$ ordre d'une autre surface réglée de la congruence $L$. Cette autre surface est confondue avec la surface de la couche (2.15) justement pour les points d'intersection de la droite (2.16) avec la conique $F$. On voit sans peine que les droites (2.16) enveloppent (si c varie) la conique (2.11). Le point de contact de la droite (2.16) avec la conique (2.11) est déterminé par la relation

$$
\begin{gathered}
a_{1}: a_{2}: a_{3}=\left(\begin{array}{c}
2 c, \beta_{2}-\alpha_{1} c^{2}, \alpha_{2}-\beta_{1} c^{2} \\
1,-\alpha_{1} c,-\beta_{1} c
\end{array}\right)= \\
=c\left(\beta_{1} \beta_{2}-\alpha_{1} \alpha_{2}\right):-\left(\alpha_{2}+c^{2} \beta_{1}\right):\left(\beta_{2}+c^{2} \alpha_{1}\right) .
\end{gathered}
$$

Si la congruence $L$ est une congruence $W$, alors les droites (2.16) passent par le point singulier (2.13) de la conique $F$.

Dorénavant, nous voulons supposer

$$
\alpha_{1} \alpha_{2}-\beta_{1} \beta_{2} \neq 0,
$$

c.-à-d. la congruence $L$ n'est pas une congruence $W$ (ce cas voir chap. 3 ).

Maintenant nous allons déterminer les complexes tangents qui contiennent le voisinage du $2^{\mathrm{e}}$ ordre des surfaces de la congruence $L$ géométriquement remarquables. Pourque le complexe tangent contienne le voisinage du $2^{\mathrm{e}}$ ordre des surfaces $\omega_{2}=$ $=c_{i} \omega_{1}(i=1,2)$, d'après $(2.8)$ ou (2.16) il est nécessaire et il suffit que

$$
c_{1}+c_{2}=\frac{2 a_{1}}{\alpha_{1} a_{2}+\beta_{1} a_{3}}, \quad c_{1} c_{2}=-\frac{\beta_{2} a_{2}+\alpha_{2} a_{3}}{\alpha_{1} a_{2}+\beta_{1} a_{3}} ;
$$

alors $a_{1}, a_{2}, a_{3}$ sont solutions du système des équations

$$
\begin{array}{r}
2 a_{1}-\alpha_{1}\left(c_{1}+c_{2}\right) a_{2}-\beta_{1}\left(c_{1}+c_{2}\right) a_{3}=0, \\
\left(\beta_{2}+\alpha_{1} c_{1} c_{2}\right) a_{2}+\left(\alpha_{2}+\beta_{1} c_{1} c_{2}\right) a_{3}=0,
\end{array}
$$

c.-à-d. on a

$$
a_{1}: a_{2}: a_{3}=\left(c_{1}+c_{2}\right)\left(\beta_{1} \beta_{2}-\alpha_{1} \alpha_{2}\right):-2\left(\alpha_{2}+\beta_{1} c_{1} c_{2}\right): 2\left(\beta_{2}+\alpha_{1} c_{1} c_{2}\right) .
$$

Nous voulons désigner ce complexe tangent par $\Omega\left[c_{1}, c_{2}\right]$ et nous avons $\Omega\left[c_{1}, c_{2}\right] \equiv$ $\equiv \Omega\left[c_{2}, c_{1}\right]$. 
Si le point $\Omega\left(a_{1}: a_{2}: a_{3}\right)\left(a_{2}, a_{3}\right.$ ne sont pas en même temps nuls) est situé sur la conique $F$, alors on obtient de (2.10) l'équation différentielle de la couche des surfaces dont le voisinage du $2^{\mathrm{e}}$ ordre est contenu dans le complexe $\Omega\left(a_{1}: a_{2}: a_{3}\right)$ sous la forme

$$
\frac{\omega_{1}}{\omega_{2}}=\frac{-a_{1}}{\beta_{2} a_{2}+\alpha_{2} a_{3}} .
$$

De-là, il résulte que les deux points d'intersection de la droite $a_{3}=0\left(a_{2}=0\right)$ avec la conique (2.11) sont les images secondaires des complexes qui contiennent le voisinage du $2^{\mathrm{e}}$ ordre de la surface réglée de la congruence $L$ (et seulement de cette surface) dont les droites sont tangentes à la première (seconde) nappe focale aux points de l'une ou de l'autre des courbes asymptotiques qui passent par le foyr $A_{1}\left(A_{2}\right)$ sur cette nappe. Ces complexes (spéciaux) sont déterminés par les relations suivantes:

$$
\text { pour } \sqrt{ }\left(\beta_{2}\right) \omega_{1}= \pm \sqrt{ }\left(-\alpha_{1}\right) \omega_{2} \text {, resp. } \sqrt{ }\left(\alpha_{2}\right) \omega_{1}= \pm \sqrt{ }\left(-\beta_{1}\right) \omega_{2} \text {, }
$$

c.-à-d. pour $c_{1,2}= \pm \sqrt{ }\left(-\beta_{2} / \alpha_{1}\right)$, resp. $c_{1,2}= \pm \sqrt{ }\left(-\alpha_{2} / \beta_{1}\right)$ on obtient de (2.19)

$$
\Omega\left[c_{1}, c_{2}\right]=\sigma\left(\sqrt{ }\left(-\alpha_{1} \beta_{2}\right)\left[A_{1} A_{2}\right] \pm\left[A_{1} A_{3}\right]\right),
$$

resp.

$$
\Omega\left[c_{1}, c_{2}\right]=\sigma\left(\sqrt{ }\left(-\beta_{1} \alpha_{2}\right)\left[A_{1} A_{2}\right] \pm\left[A_{2} A_{4}\right]\right)
$$

où $\sigma(\neq 0)$ est un facteur arbitraire.

Pour abréger, désignons par $( \pm 1)$, resp. $( \pm 2)$ la surface réglée (dans la congruence $L)$ qui est tangente à la première, resp. seconde nappe focale aux points de la courbe asymptotique $(2.25)_{1}$, resp. $(2.25)_{2}$. Soit $\Omega[j, k](j, k= \pm 1, \pm 2)$ le complexe tangent qui contient le voisinage du second ordre de la surface $(j)$ et $(k)$ (pour $j=k$ on obtient les complexes (2.26)). En se référant aux relations (2.23), on obtient les six complexes tangents suivants (les images secondaires de ces complexes sont des points d'intersection des 4 tangentes de la conique $F$ aux points $\Omega[1,1], \Omega[-1,-1], \Omega[2,2]$, $\Omega[-2,-2]$ qui sont déterminés par les relations $(2.26))$ :

$$
\begin{aligned}
\Omega[1,-1]= & \sigma\left\{\left(\alpha_{1} \alpha_{2}+\beta_{1} \beta_{2}\right)\left[A_{1} A_{3}\right]-2 \alpha_{1} \beta_{2}\left[A_{2} A_{4}\right]\right\}, \\
\Omega[2,-2]= & \sigma\left\{2 \beta_{1} \alpha_{2}\left[A_{1} A_{3}\right]-\left(\alpha_{1} \alpha_{2}+\beta_{1} \beta_{2}\right)\left[A_{2} A_{4}\right]\right\}, \\
\Omega[1,2]= & \sigma\left\{\left[\sqrt{ }\left(\alpha_{1} \alpha_{2}\right)+\sqrt{ }\left(\beta_{1} \beta_{2}\right)\right]^{2}\left[A_{1} A_{2}\right]-2 \sqrt{ }\left(-\beta_{1} \alpha_{2}\right)\left[A_{1} A_{3}\right]-\right. \\
& \left.-2 \sqrt{ }\left(-\beta_{2} \alpha_{1}\right)\left[A_{2} A_{4}\right]\right\}, \\
\Omega[1,-2]= & \sigma\left\{\left[\sqrt{ }\left(\alpha_{1} \alpha_{2}\right)-\sqrt{ }\left(\beta_{1} \beta_{2}\right)\right]^{2}\left[A_{1} A_{2}\right]+2 \sqrt{ }\left(-\beta_{1} \alpha_{2}\right)\left[A_{1} A_{3}\right]+\right. \\
& \left.+2 \sqrt{ }\left(-\beta_{2} \alpha_{1}\right)\left[A_{2} A_{4}\right]\right\}, \\
\Omega[-1,2]= & \sigma\left\{\left[\sqrt{ }\left(\alpha_{1} \alpha_{2}\right)-\sqrt{ }\left(\beta_{1} \beta_{2}\right)\right]^{2}\left[A_{1} A_{2}\right]-2 \sqrt{ }\left(-\beta_{1} \alpha_{2}\right)\left[A_{1} A_{3}\right]+\right. \\
& \left.+2 \sqrt{ }\left(-\beta_{2} \alpha_{1}\right)\left[A_{2} A_{4}\right]\right\}, \\
\Omega[-1,-2]= & \sigma\left\{\left[\sqrt{ }\left(\alpha_{1} \alpha_{2}\right)+\sqrt{ }\left(\beta_{1} \beta_{2}\right)\right]^{2}\left[A_{1} A_{2}\right]+2 \sqrt{ }\left(-\beta_{1} \alpha_{2}\right)\left[A_{1} A_{3}\right]+\right. \\
& \left.+2 \sqrt{ }\left(-\beta_{2} \alpha_{1}\right)\left[A_{2} A_{4}\right]\right\}, \\
& \sigma \neq 0, \text { arb. }
\end{aligned}
$$


Signalons que le rapport anharmonique des 4 points $(0,1,0),(0,0,1) \Omega[2,-2]$, $\Omega[1,-1]$ qui sont situés sur la droite $a_{1}=0$ est égal d'après $(2.14)$ à

$$
J=\frac{\left(\alpha_{1} \alpha_{2}+\beta_{1} \beta_{2}\right)^{2}}{4 \alpha_{1} \alpha_{2} \beta_{1} \beta_{2}}=\frac{(I+1)^{2}}{4 I}
$$

et alors il est l'invariant différentiel de la congruence L. On peut exprimer cet invariant en utilisant les formes différentielles $\varphi, \varphi^{*}, F_{1}, F_{2}$ dues à M. E. Čech, ${ }^{3}$ ) sous la forme

$$
J=\frac{\left(\varphi+\varphi^{*}\right)^{2}}{4 F_{1} F_{2}} .
$$

Désignons par $( \pm I)$, resp. $( \pm I I)$ la surface de la congruence $L$ qui est tangente à la première, resp. seconde nappe focale aux points des courbes $\sqrt{ }\left(\beta_{2}\right) \omega_{1}= \pm \sqrt{ }\left(\alpha_{1}\right) \omega_{2}$, resp. $\sqrt{ }\left(\alpha_{2}\right) \omega_{1}= \pm \sqrt{ }\left(\beta_{1}\right) \omega_{2}$. Par un calcul aisé, en utilisant la relation (2.23), nous obtenons les complexes tangents suivants qui contiennent chaque fois deux (pas nécessairement différentes) des surfaces citées:

$$
\begin{aligned}
\Omega[ \pm I, \pm I]= & \sigma\left\{ \pm \sqrt{ }\left(\beta_{2} \alpha_{1}\right)\left(\beta_{1} \beta_{2}-\alpha_{1} \alpha_{2}\right)\left[A_{1} A_{2}\right]-\left(\alpha_{1} \alpha_{2}+\beta_{1} \beta_{2}\right)\right. \\
& \left.\cdot\left[A_{1} A_{3}\right]+2 \alpha_{1} \beta_{2}\left[A_{2} A_{4}\right]\right\} \\
\Omega[ \pm I I, \pm I I]= & \sigma\left\{ \pm \sqrt{ }\left(\alpha_{2} \beta_{1}\right)\left(\beta_{1} \beta_{2}-\alpha_{1} \alpha_{2}\right)\left[A_{1} A_{2}\right]-2 \alpha_{2} \beta_{1}\left[A_{1} A_{3}\right]+\right. \\
& \left.+\left(\alpha_{1} \alpha_{2}+\beta_{1} \beta_{2}\right)\left[A_{2} A_{4}\right]\right\}, \\
\Omega[I,-I]= & \sigma\left[A_{1} A_{3}\right], \\
\Omega[I I,-I I]= & \sigma\left[A_{2} A_{4}\right], \\
\Omega[I, I I]= & \sigma\left\{\left(\alpha_{1} \alpha_{2}-\beta_{1} \beta_{2}\right)\left[A_{1} A_{2}\right]+2 \sqrt{ }\left(\alpha_{2} \beta_{1}\right)\left[A_{1} A_{3}\right]-\right. \\
& \left.-2 \sqrt{ }\left(\beta_{2} \alpha_{1}\right)\left[A_{2} A_{4}\right]\right\}, \\
\Omega[I,-I I]= & \sigma\left\{\left(\alpha_{1} \alpha_{2}-\beta_{1} \beta_{2}\right)\left[A_{1} A_{2}\right]-2 \sqrt{ }\left(\alpha_{2} \beta_{1}\right)\left[A_{1} A_{3}\right]-\right. \\
& \left.-2 \sqrt{ }\left(\beta_{2} \alpha_{1}\right)\left[A_{2} A_{4}\right]\right\}, \\
\Omega[-I, I I]= & \sigma\left\{\left(\alpha_{1} \alpha_{2}-\beta_{1} \beta_{2}\right)\left[A_{1} A_{2}\right]+2 \sqrt{ }\left(\alpha_{2} \beta_{1}\right)\left[A_{1} A_{3}\right]+\right. \\
& \left.+2 \sqrt{ }\left(\beta_{2} \alpha_{1}\right)\left[A_{2} A_{4}\right]\right\}, \\
\Omega[-I,-I I]= & \sigma\left\{\left(\alpha_{1} \alpha_{2}-\beta_{1} \beta_{2}\right)\left[A_{1} A_{2}\right]-2 \sqrt{ }\left(\alpha_{2} \beta_{1}\right)\left[A_{1} A_{3}\right]+\right. \\
& \left.+2 \sqrt{ }\left(\beta_{2} \alpha_{1}\right)\left[A_{2} A_{4}\right]\right\}, \\
& \sigma \neq 0, \operatorname{arb} .
\end{aligned}
$$

Si enfin les couches examinées sont les couches des surfaces développables de $L$, alors pour

$$
\omega_{1}^{2}=0 \quad \text { ou } \quad \omega_{2}^{2}=0 \quad \text { ou } \quad \omega_{1} \omega_{2}=0
$$

${ }^{3}$ ) On appelle les formes différentielles $\varphi=\alpha_{1} \alpha_{2} \omega_{1} \omega_{2}, q^{*}=\beta_{1} \beta_{2} \omega_{1} \omega_{2}, F_{1}=\alpha_{1} \beta_{1} \omega_{2}^{3} / \omega_{1}$, $F_{2}=\alpha_{2} \beta_{2} \omega_{1}^{3} / \omega_{2}$ par suite: la forme ponctuelle, planaire, la première et la seconde forme focale (v. [1], p. 263). 
on obtient les complexes

$$
\begin{gathered}
{ }^{1} \Omega=\sigma\left\{\beta_{1}\left[A_{1} A_{3}\right]-\alpha_{1}\left[A_{2} A_{4}\right]\right\} \quad \text { ou }{ }^{2} \Omega=\sigma\left\{\alpha_{2}\left[A_{1} A_{3}\right]-\beta_{2}\left[A_{2} A_{4}\right]\right\} \\
\text { ou }{ }^{3} \Omega=\sigma\left[A_{1} A_{2}\right], \\
\sigma \neq 0, \text { arb. }
\end{gathered}
$$

respectivement.

Les complexes tangents $(2.26),(2.27),(2.29)$ et (2.31) sont liés d'une manière invariante au voisinage différentiel du $1^{\text {er }}$ ordre de la droite $\left[A_{1} A_{2}\right]$ de la congruence $L$.

La droite $\overline{\left[A_{1} A_{3}\right]\left[A_{2} A_{4}\right]}$, c.-à-d. $a_{1}=0$, est l'image des complexes tangents qui contiennent le voisinage du $2^{\mathrm{e}}$ ordre des surfaces $\omega_{2}=c \omega_{1}$ et $\omega_{2}=-c \omega_{1}$. Les images secondaires des complexes tangents qui renferment le voisinage du $2^{\mathrm{e}}$ ordre de la surface développable $\omega_{1}=0$, resp. $\omega_{2}=0$ sont situés sur la tangente à la conique (2.11) au point ${ }^{1} \Omega$, resp. ${ }^{2} \Omega$; cette tangente passe par le $K$-point $\left[A_{1} A_{2}\right]$.

Les points ${ }^{1} \Omega$ et ${ }^{2} \Omega$ déterminés par les relations $\left.(2.31)^{4}\right)$ sont conjugués par rapport à la K-quadrique et alors les complexes ${ }^{1} \Omega$ et ${ }^{2} \Omega$ sont en involution si et seulement si on a

$$
\alpha_{1} \alpha_{2}+\beta_{1} \beta_{2}=0
$$

cela signifie: L est une congruence $V$ (c.-à-d. une congruence pour laquelle aux courbes asymptotiques sur chaque surface focale correspondent des courbes conjuguées sur l'autre surface focale (v. [2], p. 352). De là on voit aussi: si Lest une congruence $V$, alors les tangentes à la conique (2.11) passant par le $K$-point $\left[A_{1} A_{2}\right]$ et les droites $\overline{\left[A_{1} A_{2}\right]\left[A_{1} A_{3}\right]}$ et $\overline{\left[A_{1} A_{2}\right]\left[A_{2} A_{4}\right]}$ se séparent harmoniquement.

Les complexes (2.26) et (2.27) sont confondus avec les complexes (2.29) si et seulement si $L$ est une congruence $V$; dans ce cas, les substitutions suivantes sont vérifiées:

$$
\left(\begin{array}{rrrrrrrr}
I & -I & I I & -I I & 1 & -1 & 2 & -2 \\
2 & -2 & -1 & 1 & -I I & I I & I & -I
\end{array}\right) ;
$$

pour les complexes ${ }^{1} \Omega$ et ${ }^{2} \Omega$ on obtient

$$
\begin{gathered}
{ }^{1} \Omega={ }^{1} \sigma\left(\beta_{1}\left[A_{1} A_{3}\right]-\alpha_{1}\left[A_{2} A_{4}\right]\right)={ }^{1} \bar{\sigma}\left(\alpha_{2}\left[A_{1} A_{3}\right]+\beta_{2}\left[A_{2} A_{4}\right]\right), \\
{ }^{2} \Omega={ }^{2} \sigma\left(\alpha_{2}\left[A_{1} A_{3}\right]-\beta_{2}\left[A_{2} A_{4}\right]\right)={ }^{2} \bar{\sigma}\left(\beta_{1}\left[A_{1} A_{3}\right]+\alpha_{1}\left[A_{2} A_{4}\right]\right), \\
{ }^{1} \sigma,{ }^{2} \sigma,{ }^{1} \bar{\sigma},{ }^{2} \bar{\sigma} \neq 0, \text { arb. }
\end{gathered}
$$

Nous obtenons le complexe tangent $\Omega^{*}$ de la dualisation $L^{*}$ de la congruence $L$ par la transformation (1.16) et la transformation

$$
\left(\begin{array}{llll}
\Omega & a_{12} & a_{13} & a_{24} \\
\Omega^{*} & a_{34}^{*} & a_{13}^{*} & a_{24}^{*}
\end{array}\right)
$$

\footnotetext{
$\left.{ }^{4}\right)$ M. S. P. Finikov appelle les complexes ${ }^{1} \Omega$ et ${ }^{2} \Omega$ complexes associés au foyer $A_{1}$ et $A_{2}$ (сопровождающий комплекс для фокуса $A_{i}, i=1,2$ ); ces complexes sont liés d'une manière invariante au voisinage du $2^{\mathrm{e}}$ ordre de la droite $\left[A_{1} A_{2}\right]$ (v. [2], $\left.\S \S 177,202\right)$.
} 
où nous avons introduit pour un moment la notation d'une telle manière que le coefficient de $\left[A_{i} A_{j}\right]$ dans la relation (2.3) soit indiqué par $a_{i j}$ et le coefficient de $\left[E_{i} E_{j}\right]$ par $a_{i j}^{*}$.

Par les mêmes transformations on déduit pour la dualisation $L^{*}$ les relations analogues aux relations $(2.9)-(2.34)$. Comme pour les droites des repères (1.1) et (1.10) on a

$$
\left[A_{1} A_{2}\right] \equiv\left[E_{3} E_{4}\right],\left[A_{1} A_{3}\right] \equiv\left[E_{2} E_{4}\right], \quad\left[A_{2} A_{4}\right] \equiv\left[E_{1} E_{3}\right], \ldots,
$$

on voit que les points $\Omega$ et $\Omega^{*}$ de l'espace de Klein sont confondus; de même les coniques $F$ et $F^{*}$ coïncident de sorte que pour les coordonnées locales $a_{i j}$ et $a_{k l}^{*}$ résultent au fond de (2.36) les relations

$$
a_{12}=a_{34}^{*}, \quad a_{13}=a_{24}^{*}, \quad a_{24}=a_{13}^{*}, \ldots .
$$

Alors nous pouvons nous borner dans nos considérations, sans nuire en rien la généralité, à l'étude des complexes tangents de la congruence $L$, en faisant usage du repère ponctuel $(1.1)$.

3. Soit $L$ une congruence $W$ et alors la conique $F$ est singulière. Les complexes tangents de la congruence $L$ sont déterminés par la relation(2.3) et leurs images secondaires remplissent le plan (2.4). La conique $F$ se compose des droites

$$
\pm \sqrt{ }\left(-\beta_{1} / \alpha_{2}\right) a_{1}+\alpha_{1} a_{2}+\beta_{1} a_{3}=0 ;
$$

le point singulier de $F$ est donné par la relation (2.13). Les complexes $(2.31)_{1}$ et $(2.31)_{2}$ sont confondues et ils coïncident encore avec quelques uns des complexes (2.27) et (2.29).

Les droites déterminées par la relation (3.1) et les droites $\overline{\left[A_{1} A_{3}\right]\left[A_{2} A_{4}\right]}$ et $\overline{\left[A_{1} A_{2}\right]^{1} \Omega}$ (qui ont toutes le point ${ }^{1} \Omega \equiv{ }^{2} \Omega$ commun) se séparent harmoniquement parceque la droite $\overline{\left[A_{1} A_{3}\right]\left[A_{2} A_{4}\right]}$ et le point $\left[A_{1} A_{2}\right]$ sont la polaire et le pôle de la conique $F$.

Chaque point $\Omega\left(a_{1}: a_{2}: a_{3}\right)$ est l'image d'un complexe qui possède le voisinage du $2^{\mathrm{e}}$ ordre de deux surfaces réglées dont l'équation différentielle est (2.10).

A chaque surface (2.15) correspondent les $\infty^{1}$ complexes tangents de la congruence Lqui possèdent son voisinage du $2^{\mathrm{e}}$ ordre; les images secondaires de ces complexes remplissent dans le plan (2.4) une droite qui passe par le point singulier ${ }^{1} \Omega\left(0: \beta_{1}\right.$ : $\left.:-\alpha_{1}\right)$ de la conique $F$. La surface (2.15) détermine univoquement encore une autre surface réglée (dans la congruence $L$ ) dont chacun des complexes tangents mentionnés possède le voisinage du $2^{\mathrm{e}}$ ordre simultanément avec le voisinage du $2^{\mathrm{e}}$ ordre de la surface (2.15). Il y a deux cas où ces deux surfaces coïncident c.-̀̀-d. quand elles correspondent aux décompositions asymptotiques de la congruence Let alors ce sont les droites (3.1) qui forment les images secondaires de ces $\infty^{1}$ complexes tangents. On voit alors que la structure du voisinage du $2^{\mathrm{e}}$ ordre des congruences du type I qui sont ou ne sont pas $W$ est différente: si $L$ n'est pas $W$, alors à chaque couple de surfaces 
réglées arbitraires de $L$ il correspond justement un complexe tangent qui possède leurs voisinage du $2^{\mathrm{e}}$ ordre; mais si $L$ est une congruence $W$, on peut choisir dans $L$ seulement une surface arbitrairement et l'autre surface dont le voisinage du $2^{\mathrm{e}}$ ordre le même complexe tangent contient est déterminée univoquement.

Nous allons prouver l'assertion précédente. Le point arbitraire $\Omega\left(a_{1}: a_{2}: a_{3}\right)$ de la droite qui joint le point fixe $\Omega\left(\bar{a}_{1}: \bar{a}_{2}: \bar{a}_{3}\right)$ et le point $\Omega\left(0: \beta_{1}:-\alpha_{1}\right)$ est déterminé par les relations

$$
a_{1}=\lambda \bar{a}_{1}, \quad a_{2}=\lambda \bar{a}_{2}+\mu \beta_{1}, \quad a_{3}=\lambda \bar{a}_{3}-\mu \alpha_{1}, \quad \lambda \neq 0, \mu \text { arb. }
$$

En portant $a_{1}, a_{2}, a_{3}$ des relations précedentes dans $(2.10)$ on obtient $\left(\alpha_{1} \alpha_{2}-\right.$ $\left.-\beta_{1} \beta_{2}=0\right)$

$$
\left(\frac{\omega_{1}}{\omega_{2}}\right)_{1,2}=\frac{-\bar{a}_{1}^{2} \pm \sqrt{ }\left[\bar{a}_{1}^{2}+\left(\alpha_{1} \bar{a}_{2}+\beta_{1} \bar{a}_{3}\right)\left(\beta_{2} \bar{a}_{2}+\alpha_{2} \bar{a}_{3}\right)\right]}{\beta_{2} \bar{a}_{2}+\alpha_{2} \bar{a}_{3}}
$$

et comme ce rapport $\omega_{1}: \omega_{2}$ ne dépend pas des paramètres $\lambda$ et $\mu$, alors à chaque point de la droite examinée correspondent les mêmes deux surfaces de la congruence $L$ comme au point $\Omega\left(\bar{a}_{1}: \bar{a}_{2}: \bar{a}_{3}\right)$. Soit maintenant une de ces surfaces déterminée par l'équation (2.15); alors on peut choisir au lieu du point $\Omega\left(\bar{a}_{1}: \bar{a}_{2}: \bar{a}_{3}\right)$ un point arbitraire de la droite (2.16) qui passe par le point ${ }^{1} \Omega$, p. ex. $\bar{a}_{1}=\alpha_{1} c^{2}-\beta_{2}, \bar{a}_{2}=2 c$, $\bar{a}_{3}=0$ et ensuite on obtient de (3.3)

$$
\left(\frac{\omega_{2}}{\omega_{1}}\right)_{1}=c,\left(\frac{\omega_{2}}{\omega_{1}}\right)_{2}=-\frac{\beta_{2}}{\alpha_{1} c} .
$$

Les racines (3.4) coïncident si et seulement si on a

$$
c^{2}=-\frac{\beta_{2}}{\alpha_{1}}=-\frac{\alpha_{2}}{\beta_{1}},
$$

c.-à-d. pour les décompositions asymptotiques. En substituant d'après (3.5) dans l'équation (2.16) on obtient juste les droites (3.1). La preuve est donc terminée.

Comme pour les congruences $W$ les asymptotiques ainsi que les courbes dont les tangentes séparent harmoniquemant les tangentes asymptotiques se correspondent, on: obtient au lieu de 8 complexes (2.26) et (2.27) et 8 complexes (2.29) chaquefois seulement 3 complexes différents. Les droites (3.1) sont des images des $\infty^{1}$ complexes $\Omega[1,1]$ et $\Omega[-1,-1]$.

La droite $\overline{\left[A_{1} A_{3}\right]\left[A_{2} A_{4}\right]}$ est l'image des $\infty^{1}$ complexes tangents qui possèdent le voisinage du $2^{\mathrm{e}}$ ordre des surfaces $\omega_{2}= \pm \sqrt{ }\left(\beta_{1} / \alpha_{2}\right) \omega_{1}$ et la droite $\overline{\left[A_{1} A_{2}\right]^{1} \Omega}$ est l'image des complexes tangents qui possèdent le voisinage du $2^{\mathrm{e}}$ ordre des surfaces développables $\omega_{1} \omega_{2}=0$ comme il résulte de la relation (2.8) pour un point arbitraire $a_{1}=\lambda, a_{2}=\mu \beta_{1}, a_{3}=-\mu \alpha_{1}$ de cette droite.

Les complexes $\Omega[1,-1]$ et $\Omega[ \pm I, \pm I]$ coïncident avec le complexe osculateur ${ }^{1} \Omega \equiv{ }^{2} \Omega$ qui contient tout le voisinage du $2^{\mathrm{e}}$ ordre de la droite $\left[A_{1} A_{2}\right]$ et qui est le complexe osculateur de $L$. Les images secondaires des complexes ${ }^{1} \Omega$ décrivent dans 
l'espace de Klein la surface $\left({ }^{1} \Omega\right)$ qui possède un réseau conjugué et le plan $(2.4)$ est tangent à cette surface; l'auteur a fait l'étude des congruences $W$ à l'aide de la surface $\left({ }^{i} \Omega\right)$ dans le Mémoire [3] et [4].

4. Dans les chapîtres 2 et 3 nous avons étudié du point de vue des complexes tangents la structure du voisinage du $1^{\text {er }}$ et $2^{\mathrm{e}}$ ordre d'une droite fixe des congruences du type I. Dans ce chapitre, nous voulons signaler sommairement les résultats relatifs aux autres types de congruences.

Si Lest une congruence du type II, II*, V ou $\mathrm{V}^{*}$ respectivement, alors la conique $F$ est régulière et son équation résulte si l'on pose nul les invariants relatifs correspondants $\alpha_{i}$ et $\beta_{i}(i=1,2)$ dans l'équation (2.11). Si $L$ est une congruence du type II ou II*, alors la conique $F$ est tangente à la droite $\overline{\left[A_{1} A_{2}\right]\left[A_{1} A_{3}\right]}$ ou $\overline{\left[A_{1} A_{2}\right]\left[A_{2} A_{4}\right]}$ dans le $K$-point $\left[A_{1} A_{3}\right]$ ou $\left[A_{2} A_{4}\right]$; si $L$ est une congruence du type $\mathrm{V}$ ou $\mathrm{V}^{*}$, alors $F$ est tangente en même temps aux deux droites précédentes dans les deux points mentionnés. La structure du voisinage du $1^{\text {er }}$ ordre d'une droite arbitraire des congruences des types considérés est essentiellement la même que celle des congruences du type I $\left(\alpha_{1} \alpha_{2}-\beta_{1} \beta_{2} \neq 0\right)$. On peut définir tous les complexes (2.26), (2.27) et (2.29) dont quelques-uns coïncident ou dégénèrent en des complexes spéciaux. Tous deux points différents dans le plan (2.4) sont des images secondaires de deux complexes tangents dont chacun contient le voisinage du second ordre des deux couples différents des surfaces réglées de la congruence $L$. Les complexes qui contiennent le voisinage du second ordre de la surface développable dégénerée en un faisceau de droites sont spéciaux.

Les congruences du type III: une des surfaces focales dégénère en une droite qui est la directrice du complexe spécial auquel la congruence appartient. Nous voulons nous borner au type $\alpha_{1} \beta_{2} \neq 0=\alpha_{2}=\beta_{1}$ où la droite $\left[A_{2} A_{4}\right]$ est fixe. La conique $F$, donnée par l'équation

$$
a_{1}^{2}+\alpha_{1} \beta_{2} a_{2}^{2}=0
$$

est dégénerée et son point singulier est le $K$-point $\left[A_{2} A_{4}\right]$. Le point $\left[A_{1} A_{2}\right]$ et la droite $\overline{\left[A_{1} A_{3}\right]\left[A_{2} A_{4}\right]}$ sont le pôle et la droite polaire de la conique (4.1). On peut alors regarder les congruences du type III comme des congruences $W$ dégénerées et alors leurs droites poss èdent la même structure de leurs voisinages différentiels comme les congruences $W$. Le théorème signalé dans le chap. 3 est vrai et le complexe $\left[A_{2} A_{4}\right]$ spécial, auquel la congruence appartient, joue le rôle du complexe osculateur ${ }^{1} \Omega$.

Pour les congruences des type IV, VI et VI* la conique $F$ dégénère en la droite $\overline{\left[A_{1} A_{3}\right]\left[A_{2} A_{4}\right]}$ dont l'équation est $a_{1}^{2}=0$.

Les congruences $d u$ type IV $\left(\alpha_{1} \beta_{1} \neq 0=\alpha_{2}=\beta_{2}\right)$. La substitution $\alpha_{2}=\beta_{2}=0$ dans l'équation (2.8) donne

$$
\left[2 a_{1} \omega_{1}-\left(a_{2} \alpha_{1}+a_{3} \beta_{1}\right) \omega_{2}\right] \omega_{2}=0 .
$$

Chaque complexe $\Omega\left(a_{1}: a_{2}: a_{3}\right)$ contient le voisinage du $2^{\mathrm{e}}$ ordre de deux surfaces de la congruence $L$; une de ces surfaces est constamment la surface développable 
$\omega_{2}=0$ qui dégénère en un faisceau de droites dans le plan $\left[A_{1} A_{2} A_{3}\right]-$ le sommet de ce faisceau est le point $A_{2}$ situé sur la courbe directrice $\left(A_{2}\right)$. Le point ${ }^{1} \Omega\left(0: \beta_{1}:-\alpha_{1}\right)$ est l'image secondaire du complexe osculateur $\left({ }^{2} \Omega\right.$ n'est pas défini). Les points des droites qui passent par le point ${ }^{1} \Omega$ sont les images des $\infty^{1}$ complexes tangents dont chacun contient le voisinage du $2^{\mathrm{e}}$ ordre d'une même couple de surfaces de la congruence $L$; une de ces surfaces est toujours la surface $\omega_{2}=0$. Les surfaces $\omega_{2}=0$ forment l'unique décomposition asymptotique de la congruence $L$ et les images des $\infty^{1}$ complexes tangents qui contiennent le voisinage du $2^{\mathrm{e}}$ ordre de la surface $\omega_{2}=0$ (et seulement de cette surface) sont situés sur la droite $a_{1}=0$. D'après [3], chap. 3, la droite $\overline{\left[A_{1} A_{3}\right]\left[\overline{\left.A_{2} A_{4}\right]}\right.}$ (c.-à-d. $\left.a_{1}=0\right)$ est l'image des complexes osculateurs des droites de $L$ qui ont le foyer $A_{2}$ commun.

Les congruences du type VI et VI* appartiennent à un complexe linéaire spécial et alors on peut, dans un certain sens, les régarder comme congruences dégénerées du type I ou soit la surface développable soit la courbe focale dégénèrent en une droite. En se bornant au type VI $\left(\beta_{1} \neq 0=\alpha_{1}=\alpha_{2}=\beta_{2}\right)$, resp. VI* $\left(\alpha_{1} \neq 0=\alpha_{2}=\beta_{1}=\right.$ $\left.=\beta_{2}\right)$, nous obtenons au lieu de (4.2) la relation

$$
\left(2 a_{1} \omega_{1}-a_{3} \beta_{1} \omega_{2}\right) \omega_{2}=0 \text {, resp. }\left(2 a_{1} \omega_{1}-a_{2} \alpha_{1} \omega_{2}\right) \omega_{2}=0 .
$$

La structure du voisinage du $1^{\text {er }}$ ordre de ces congruences et des congruences du type IV est essentiellement la même; le complexe linéaire spécial $\left[A_{1} A_{3}\right]$, resp. $\left[A_{2} A_{4}\right]$ auquel la congruence $L$ appartient joue maintenant le rôle du complexe osculateur.

Les congruences du type VII (les congruences linéaires): Chacun des $\infty^{1}$ complexes dont les images sont situées sur la droite $a_{1}=0$ contient le voisinage du second ordre d'une droite arbitraire de la congruence et l'on voit sans peine qu'il contient toute la congruence. Les complexes $\Omega\left(a_{1}: a_{2}: a_{3}\right)$ où $a_{1} \neq 0$ ne contiennent que le voisinage du second ordre des surfaces développables $\omega_{1}=0$ et $\omega_{2}=0$ qui dégénèrent en des faisceaux de droites dans les plans $\left[A_{1} A_{2} A_{3}\right]$ et $\left[A_{1} A_{2} A_{4}\right]$; les sommets de ces faisceau sont les points $A_{2}$ et $A_{1}$.

5. Soit $L$ une congruence du type I $\left(\alpha_{1} \alpha_{2}-\beta_{1} \beta_{2} \neq 0\right)$. L'ensemble des images secondaires des complexes tangents $\Omega$ dépend de 3 paramètres homogènes $a_{1}, a_{2}, a_{3}$ et de 2 paramètres principaux des droites de la congruence $L$. Les points $\Omega$ remplissent alors dans l'espace de Klein une variété ponctuelle à quatre dimensions que nous voulons désigner $\operatorname{par}(\Omega)$. On peut aussi considérer la variété $(\Omega)$ comme une variété planaire $\Lambda$ qui est composée de $\infty^{2}$ plans (2.4) c.-à-d. comme une congruence de plans. ${ }^{5}$ ) Les points de chaque plan de la congruence $\Lambda$ sont des images de tous les complexes tangents d'une même droite de la congruence $L$ et alors les plans de la congruence $\Lambda$ et les droites de la congruence $L$ sont dans une correspondance biunivoque et le point du contact avec la K-quadrique (hyperquadrique de Klein) d'un plan arbitraire de la congruence $\Lambda$ est l'image de Klein de la droite correspondante de la congruence $L$.

$\left.{ }^{5}\right)$ Dans l'espace á cinq dimensions de Klein nous voulons entendre sous le nom de congruence d'espaces linéaires $\bar{P}_{i}(0<i \leqq 4)$ l'ensemble de ces espaces qui dépend de deux paramètres. 
Nous allons déterminer les points singuliers (les foyers) et les directions focales de la congruence $\Lambda$; le point $\Omega$ est nommé foyer du plan $\bar{P}_{2}$ de la congruence $\Lambda$ si pour un certain déplacement de ce plan dans la direction $\omega_{2}: \omega_{1}$ - direction focale - le point $\mathrm{d} \Omega$ est situé dans le plan $\Lambda$ (voir C. SERGE: Preliminari di una teoria delle varieta luoghi di spazi, Rend. Palermo, t. XXX, 1910, С. П. Фиников: Теория пар конгруэнций, Москва, 1956, chap. 25).

Pour le complexe tangent (2.3) de la droite $\left[A_{1} A_{2}\right]$ de la congruence $L$ on a

$$
\begin{aligned}
\mathrm{d} \Omega= & {\left[\mathrm{d} a_{1}+a_{1}\left(\omega_{11}+\omega_{22}\right)+a_{2} \omega_{32}-a_{3} \omega_{41}\right]\left[A_{1} A_{2}\right]+} \\
& +\left[\mathrm{d} a_{2}+a_{2}\left(\omega_{11}+\omega_{33}\right)\right]\left[A_{1} A_{3}\right]+\left[\mathrm{d} a_{3}+a_{3}\left(\omega_{22}+\omega_{44}\right)\right] . \\
& \cdot\left[A_{2} A_{4}\right]+\left(a_{1} \omega_{2}+a_{2} \beta_{2} \omega_{1}+a_{3} \alpha_{2} \omega_{1}\right)\left[A_{1} A_{4}\right]- \\
& -\left(a_{1} \omega_{1}-a_{2} \alpha_{1} \omega_{2}-a_{3} \beta_{1} \omega_{2}\right)\left[A_{2} A_{3}\right] .
\end{aligned}
$$

Les points $\mathrm{d} \Omega$ remplissent en général dans l'espace de Klein un espace à quatre dimensions

$$
\bar{P}_{4} \equiv\left\{\left[A_{1} A_{2}\right],\left[A_{1} A_{3}\right],\left[A_{2} A_{4}\right],\left[A_{1} A_{4}\right],\left[A_{2} A_{3}\right]\right\}
$$

qui est l'espace tangent de la variété $(\Omega)$ dans le point $\Omega$; cet espace passe par les plans (2.4) et (2.5) et est en même temps l'espace tangent de la $K$-quadrique en le $K$-point $\left[A_{1} A_{2}\right]$. Les espaces tangents $(5.2)$ de la variété $(\Omega)$ dans les points $\Omega$ d'un même plan (2.4) sont identiques parceque les paramètres pricipaux (mais non leurs différentielles) dont le répère (1.1) dépend possèdent toujours les mêmes valeurs et alors les points qui déterminent l'espace (5.2) sont fixes.

La condition nécessaire et suffisante pour que le point $\mathrm{d} \Omega$ soit situé dans le plan (2.4) est d'avoir

$$
\begin{aligned}
a_{1} \omega_{1}-\left(a_{2} \alpha_{1}+a_{3} \beta_{1}\right) \omega_{2} & =0, \\
\left(a_{2} \beta_{2}+a_{3} \alpha_{2}\right) \omega_{1}+a_{1} \omega_{2} & =0 .
\end{aligned}
$$

En général, ces relations ne sont pas remplies identiquement pour $a_{1}, a_{2}, a_{3}$ arbitraires; car on aurait nécessairement $\omega_{1}=\omega_{2}=0\left(\alpha_{1} \alpha_{2} \beta_{1} \beta_{2} \neq 0\right)$ et alors la droite de la congruence $L$ serait fixe et il s'agirait seulement d'un mouvement dans l'ensemble des complexes tangents le long d'une droite fixe.

Si les équations (5.3) ne sont pas remplies identiquement pour $a_{1}, a_{2}, a_{3}$ arbitraires, alors la condition nécessaire et suffisante pour qu'elles déterminent le rapport $\omega_{2}: \omega_{1}$ est: le déterminant de ce système est nul, c.-à-d. les points singuliers de la congruence $A$ sont situés sur la conique $F$ (déterminée par la relation (2.11)) qui est alors la courbe focale dans le plan (2.4) de la congruence $\Lambda$.

Si les relations (5.3) sont remplies identiquement pour $\omega_{2}$ et $\omega_{1}$ arbitraires, alors on a nécessairement $\alpha_{1} \alpha_{2}-\beta_{1} \beta_{2}=0$ et $L$ est une congruence $W$. Supposons pour un moment que $L$ est une congruence $W$. Alors le point $a_{1}=0, a_{2}: a_{3}=-\alpha_{2}: \beta_{2}$ $\left(=-\beta_{1}: \alpha_{1}\right)$ est le foyer pour lequel, d'après la définition de ci-dessus, toute direction $\omega_{1}: \omega_{2}$ est une direction focale. Tous les points $\Omega$, sur chacune des droites (3.1) dont la conique $F$ se compose, possèdent les directions focales égales; ces directions cor- 
respondent aux décompositions asymptotiques de la congruence $L$ comme il résulte facilement si nous substituons à $\alpha_{1} a_{2}+\beta_{1} a_{3}$ d'après (3.1) dans la relation $(5.3)_{2}$.

Supposons de nouveau $\alpha_{1} \alpha_{2}-\beta_{1} \beta_{2} \neq 0$. Les coordonnées d'un point arbitraire $\Omega$ de la conique $F$ sont déterminées par les relations (2.19); en portant de là $a_{1}, a_{2}, a_{3}$ dans une des équations (5.3), on obtient (au facteur $c\left(\beta_{1} \beta_{2}-\alpha_{1} \alpha_{2}\right)$ non nul près) l'équation différentielle (2.15) qui détermine alors les directions focales dans le point $\Omega$ considéré. La relation (5.1) donne pour les mêmes valeurs $a_{1}, a_{2}, a_{3}$ :

$$
\begin{aligned}
\mathrm{d} \Omega= & \left\{\mathrm{d}\left[c\left(\beta_{1} \beta_{2}-\alpha_{1} \alpha_{2}\right)\right]+c\left(\beta_{1} \beta_{2}-\alpha_{1} \alpha_{2}\right)\left(\omega_{11}+\omega_{22}\right)-\right. \\
& \left.-\left(\alpha_{2}+c^{2} \beta_{1}\right) \omega_{32}-\left(\beta_{2}+c^{2} \alpha_{1}\right) \omega_{41}\right\}\left[A_{1} A_{2}\right]- \\
& -\left\{\mathrm{d}\left(\alpha_{2}+c^{2} \beta_{1}\right)+\left(\alpha_{2}+c^{2} \beta_{1}\right)\left(\omega_{11}+\omega_{33}\right)\right\}\left[A_{1} A_{3}\right]+ \\
& +\left\{\mathrm{d}\left(\beta_{2}+c^{2} \alpha_{1}\right)+\left(\beta_{2}+c^{2} \alpha_{1}\right)\left(\omega_{22}+\omega_{44}\right)\right\}\left[A_{2} A_{4}\right]+ \\
& \left.+\left(\beta_{1} \beta_{2}-\alpha_{1} \alpha_{2}\right)\left(\omega_{2}-c \omega_{1}\right)\left(c\left[A_{1} A_{4}\right]+\left[A_{2} A_{3}\right]\right),{ }^{6}\right)
\end{aligned}
$$

alors i'espace tangent dans le point (2.19) de la variété $(\Omega)$ est un espace à trois dimensions. On a donc l'énoncé suivant:

Les points singuliers de la congruence $\Lambda$ remplissent dans l'espace de Klein une variété ponctuelle $(F)$ à trois dimensions qui possède $\infty^{2}$ de coniques situées dans les $\infty^{2}$ plans (2.4). Les plans (2.4) des images secondaires des complexes tangents le long des droites de la surface (2.15) de la congruence L déterminent un système à un paramètre de plans de la congruence $\Lambda$; tous deux plans consécoutifs de ce système possèdent justement un point commun donné par les relations (2.19). Ce point est situé sur les coniques $F$ correspondantes et décrit sur la variété $(F)$ une courbe dont l'équation différentielle est (2.15).

On peut prouver l'assertion que deux plans consécoutifs possèdent justement un point commun de la manière suivante. La matrice

$$
\begin{aligned}
& \left\{\left[A_{1} A_{2}\right],\left[A_{1} A_{3}\right],\left[A_{2} A_{4}\right], \mathrm{d}\left[A_{1} A_{2}\right], \mathrm{d}\left[A_{1} A_{3}\right], \mathrm{d}\left[A_{2} A_{4}\right]\right\}= \\
=\left\{\left[A_{1} A_{2}\right],\right. & {\left[A_{1} A_{3}\right],\left[A_{2} A_{4}\right], \omega_{2}\left[A_{1} A_{4}\right]-\omega_{1}\left[A_{2} A_{3}\right], \beta_{2} \omega_{1}\left[A_{1} A_{4}\right]+} \\
& \left.+\alpha_{1} \omega_{2}\left[A_{2} A_{3}\right], \alpha_{1} \omega_{1}\left[A_{1} A_{4}\right]+\beta_{1} \omega_{2}\left[A_{2} A_{3}\right]\right\}
\end{aligned}
$$

est en yénéral du rang 5 , c.-à-d. deux plans infinement voisins $\bar{P}_{2}$ sont situés dans un espace $\bar{P}_{4}$ et possèdent alors au moins un point commun. La condition nécessaire et suffisante pourque ces deux espaces se coupent dans une droite est: le rang de la matrice $(5.5)$ est 4 , c.-à-d. on a

$$
-\frac{\omega_{2}}{\omega_{1}}=\frac{\beta_{2} \omega_{1}}{\alpha_{1} \omega_{2}}=\frac{\alpha_{2} \omega_{1}}{\beta_{1} \omega_{2}}
$$

de-là on voit que la congruence $L$ doit être nécessairement une congruence $W$ et les directions focales correspondent aux décompositions asymptotiques de $L$.

$\left.{ }^{6}\right)$ On ne peut pas conclure de cette relation que pour les congruences $W\left(\alpha_{1} \alpha_{2}-\beta_{1} \beta_{2}=0\right)$ toutes les directions dans les points de la conique $F$ soient des directions focales; si $L$ est une congruence $W$, alors les coordonnées d'un point arbitraire de la conique $F$ ne sont pas déterminées par les relations (2.19). 
D'après le lemme de Cartan, on obtient des relations (1.8):

$$
\begin{aligned}
\omega_{32} & =\gamma_{1} \omega_{1}+\gamma_{2} \omega_{2}, \\
\omega_{41} & =\gamma_{3} \omega_{1}+\gamma_{4} \omega_{2}, \\
\mathrm{~d} \alpha_{1}+\alpha_{1}\left(2 \omega_{22}-\omega_{11}-\omega_{44}\right) & =\gamma_{2} \omega_{1}+\gamma_{5} \omega_{2}, \\
\mathrm{~d} \alpha_{2}+\alpha_{2}\left(2 \omega_{11}-\omega_{22}-\omega_{33}\right) & =\gamma_{6} \omega_{1}+\gamma_{3} \omega_{2}, \\
\mathrm{~d} \beta_{1}+\beta_{1}\left(\omega_{22}+\omega_{33}-2 \omega_{44}\right) & =-\gamma_{4} \omega_{1}+\gamma_{7} \omega_{2}, \\
\mathrm{~d} \beta_{2}+\beta_{2}\left(\omega_{11}+\omega_{44}-2 \omega_{33}\right) & =\gamma_{8} \omega_{1}-\gamma_{1} \omega_{2} .
\end{aligned}
$$

La différentiation extérieure de la relation (2.15) entraîne

$$
\left[\mathrm{d} c+c\left(\omega_{11}-\omega_{33}-\omega_{22}+\omega_{44}\right) \omega_{1}\right]=0
$$

et alors on $\mathrm{a}\left(\omega_{11}+\omega_{22}+\omega_{33}+\omega_{44}=0\right)$

$$
\mathrm{d} c+2 c\left(\omega_{11}+\omega_{44}\right)=\gamma \omega_{1} .
$$

On voit de la relation (5.4) que pour $\omega_{2}=c \omega_{1}$ le point $\mathrm{d} \Omega$ est situé dans le plan (2.4). Si $\mathrm{d}_{1}$ signifie la différentiation sous la supposition $\omega_{2}=c \omega_{1}$ alors en utilisant les relations (5.7) et (5.9) on obtient de (5.4)

$$
\begin{aligned}
\mathrm{d}_{1} \Omega & =\left(b_{1}\left[A_{1} A_{2}\right]+b_{2}\left[A_{1} A_{3}\right]+b_{3}\left[A_{2} A_{4}\right]\right) \omega_{1}- \\
& -\left(\omega_{11}\left(\mathrm{~d}_{1}\right)-\omega_{22}\left(\mathrm{~d}_{1}\right)-2 \omega_{33}\left(\mathrm{~d}_{1}\right)\right) \Omega,
\end{aligned}
$$

où

$$
\begin{aligned}
b_{1}= & \left(\beta_{1} \beta_{2}-\alpha_{1} \alpha_{2}\right) \gamma-\left(\alpha_{2}+2 \beta_{1} c^{2}\right) \gamma_{1}-c\left(2 \alpha_{2}+\beta_{1} c^{2}\right) \gamma_{2}- \\
& -\left(\beta_{2}+2 \alpha_{1} c^{2}\right) \gamma_{3}-c\left(2 \beta_{2}+\alpha_{1} c^{2}\right) \gamma_{4}-\alpha_{2} c^{2} \gamma_{5}-\alpha_{1} c \gamma_{6}+ \\
& +\beta_{2} c^{2} \gamma_{7}+\beta_{1} c \gamma_{8}, \\
-b_{2}= & 2 c \beta_{1} \gamma+c \gamma_{3}-c^{2} \gamma_{4}+\gamma_{6}+c^{3} \gamma_{7}, \\
b_{3}= & 2 c \alpha_{1} \gamma-c \gamma_{1}+c^{2} \gamma_{2}+c^{3} \gamma_{5}+\gamma_{8} .
\end{aligned}
$$

De (5.10) il s'ensuit

$$
\begin{aligned}
\mathrm{d}_{1}^{2} \Omega= & \left(b_{1} c+\beta_{2} b_{2}+\alpha_{2} b_{3}\right) \omega_{1}\left[A_{1} A_{4}\right]+\left(-b_{1}+\alpha_{1} c b_{2}+\beta_{1} c b_{3}\right) \\
& . \omega_{1}\left[A_{2} A_{3}\right]+\text { comb. lin. de }\left(\left[A_{1} A_{2}\right],\left[A_{1}, A_{3}\right],\left[A_{2} A_{4}\right]\right)
\end{aligned}
$$

Le plan (2.4) est un plan tangent à la courbe (2.15) de la variété $(F)$, mais en général il n'est pas son plan osculateur. ${ }^{7}$ )

${ }^{7}$ ) Car cela étant, les relations $c b_{1}+\beta_{2} b_{2}+\alpha_{2} b_{3}=0, b_{1}-\alpha_{1} c b_{2}-\beta_{1} c b_{3}=0$ (qui sont les polynômes de 4 degré en $c$ ) seraient vraies pour $c$ arbitraire et alors tous les coefficients des puissances de $c$ seraient nuls. En supposant que $\gamma$ et $\gamma_{i}(i=1,2, \ldots, 8)$ ne sont pas nuls (le cas le plus général), on obtient en annulant les coefficients de $c^{4}$ et $c$ dans la première et la seconde équation les relations $\beta_{1} \gamma_{2}+\alpha_{1} \gamma_{4}=0$ et $\alpha_{2} \gamma_{2}+\beta_{2} \gamma_{4}=0$; il en résulte $\gamma_{2} / \gamma_{4}=-\alpha_{1} / \beta_{1}=$ $=-\beta_{2} / \alpha_{2}$ et alors $\alpha_{1} \alpha_{2}-\beta_{1} \beta_{2}=0$, ce qui est en contradiction avec la supposition $\alpha_{1} \alpha_{2}-$ $-\beta_{1} \beta_{2} \neq 0$.

Si les relations précitées ne sont pas identiquement nulles, elles peuvent posséder seulement un nombre fini de racines communnes et pour une telle racine $c$ nous avons $b_{1}: b_{2}: b_{3}=c\left(\beta_{1} \beta_{2}-\right.$ $\left.-\alpha_{1} \alpha_{2}\right):-\left(\alpha_{2}+c^{2} \beta_{1}\right):\left(\beta_{2}+c^{2} \alpha_{1}\right)$ et alors $\mathrm{d}_{1} \Omega=(.) \Omega$ de sorte que le point $\Omega$ est fixe; pour un $c$ de cette propriété chaque surface réglée de la couche $\omega_{2}=c \omega_{1}$ appartient à un complexe linéaire fixe. Si telles couches existent c.-à-d. si le résultant des équations énoncées de 4 dégré est nul, il n'en existe que 4 au maximum. 
La condition nécessaire et suffisante pour que la droite $\left\{\Omega, \mathrm{d}_{1} \Omega\right\}$ soit tangente à la conique $F$ dans le plan (2.4) est qu'on ait

$$
2 a_{1} b_{1}+2 \alpha_{1} \beta_{2} a_{2} b_{2}+\left(\alpha_{1} \alpha_{2}+\beta_{1} \beta_{2}\right)\left(a_{2} b_{3}+b_{2} a_{3}\right)+2 \beta_{1} \alpha_{2} a_{3} b_{3}=0
$$

où $a_{i}$ et $b_{i}$ sont déterminées par les relations (2.19) et (5.11); la substitution donne (5.13)' $\left(\alpha_{1} \gamma_{7}-\beta_{1} \gamma_{5}\right) c^{5}-3\left(\gamma_{2} \beta_{1}+\gamma_{4} \alpha_{1}\right) c^{4}-\left(3 \gamma_{1} \beta_{1}+3 \alpha_{1} \gamma_{3}+\alpha_{2} \gamma_{5}-\beta_{2} \gamma_{7}\right) c^{3}-$ $-\left(3 \alpha_{2} \gamma_{2}+3 \beta_{2} \gamma_{4}+\alpha_{1} \gamma_{6}-\beta_{1} \gamma_{8}\right) c^{2}-3\left(\alpha_{2} \gamma_{1}+\beta_{2} \gamma_{3}\right) c-\left(\beta_{2} \gamma_{6}-\alpha_{2} \gamma_{8}\right)=0$.

En général cette équation n'est pas vérifiée pour $c$ arbitraire. Si on exclut le cas mentionné, on voit que sur chaque conique $F$ (tant qu'on $a \alpha_{1} \gamma_{7}-\beta_{1} \gamma_{5} \neq 0$ ) il existe cinq points qui décrivent sur la variété $(F)$ cinq courbes qui sont tangentes à la conique $F$ en chaque point correspondant.

Déterminons maintenant la signification géométrique des courbes $\omega_{2}=c \omega_{1}$ sur la variété $(F)$ pour la congruence $L$. Les points de la courbe $\omega_{2}=c \omega_{1}$ sur la variété $(F)$ sont des images secondaires d'un système à un paramètre de complexes linéaires; cette courbe détermine alors dans l'espace $P_{3}$ une congruence $W$ de Segre si les plans tangents (2.4) de cette courbe ne sont pas leurs plans osculateurs - dorénavant, nous voulons exclure ces courbes de nos considérations. Les tangentes des courbes (2.15) coupent la $K$-quadrique aux $K$-points

$$
\begin{aligned}
& X_{1}=\left(a_{1} b_{3}-b_{1} a_{3}\right)\left[A_{1} A_{2}\right]+\left(a_{2} b_{3}-a_{3} b_{2}\right)\left[A_{1} A_{3}\right] \\
& X_{2}=\left(a_{1} b_{2}-b_{1} a_{2}\right)\left[A_{1} A_{2}\right]-\left(a_{2} b_{3}-a_{3} b_{2}\right)\left[A_{2} A_{4}\right]
\end{aligned}
$$

$\left(a_{i}\right.$ et $b_{i}$ sont déterminés par les relations (2.19) et (5.11)); ces $K$-points sont les images de Klein des droites de l'espace $P_{3}$ situées dans le premier, resp. second plan focal de la congruence $L$ envisagée et tangentes à la première surface focale en le foyer $A_{1}$, resp. seconde surface focale en le foyer $A_{2}$. Les droites $X_{1}$ et $X_{2}$ décrivent dans l'espace $P_{3}$ deux surfaces réglées tangentes aux surfaces focales de la congruence $L$ aux points des courbes $\omega_{2}=c \omega_{1}$; ces surfaces réglées sont les nappes focales d'une congruence de Segre que nous voulons nommer la congruence $W$ de Segre tangente à la congruence $L .^{8}$ )

Dorénavant, nous voulons exclure de nos considérations les congruences $W$. L'équation différentielle

$$
\omega_{2}=c_{1} \omega_{1}, \quad c_{1} \neq 0
$$

détermine une décomposition de la congruence $L$ à une couche de surfaces. Les images secondaires des complexes tangents qui possèdent le voisinage du second ordre des surfaces de la couche (5.15) remplissent dans l'espace $\bar{P}_{5}$ une congruence de droites; chaque droite de cette congruence est située juste dans un plan de la congruence $\Lambda$ et elle est tangente à la conique focale $F$ dans ce plan. Nous voulons désigner cette congruence par $\Omega\left(c_{1}\right)$. Déterminons les foyers de cette congruence. La relation (2.18), resp. (2.16), détermine dans le plan (2.4) pour $c=c_{1}$ la droite de la congruence $\Omega\left(c_{1}\right)$. La

\footnotetext{
$\left.{ }^{8}\right)$ La notion des congruences tangentes de Segre pour les congruences $W$ l'auteur a introduit dans le Mémoire [3].
} 
condition nécessaire et suffisante pour que la droite (2.18) de cette congruence possède un foyer est que pour un certain rapport $\lambda: \mu$ le point $\mathrm{d} \Omega$ soit situé sur cette droite. Alors il est nécessaire que dans la relation (5.1) les coefficients de $\left[A_{1} A_{4}\right]$ et $\left[A_{2} A_{3}\right]$ (où $a_{1}, a_{2}, a_{3}$ sont donnés par les relations (2.17)) soient nuls; il en résulte s'il existe sur la droite (2.18) un foyer il n'y en est qu'un et c'est le point du contact de la droite (2.18) avec la conique $F$. Si nous désignons ce foyer par $\Omega$ alors nécessairement les points $\Omega$ et $\mathrm{d} \Omega$ sont conjugués par rapport à la conique $F$ et alors $c_{1}$ est la racine de l'équation (5.13).

Choisissons dans chaque plan de la congruence $\Lambda$ un point, p. ex. de la manière suivante: nous décomposons la congruence $L$ en deux couches de surfaces réglées qui sont déterminées par l'équation différentielle (5.15) et l'équation différentielle

$$
\omega_{2}=c_{2} \omega_{1}, \quad c_{2} \neq 0 ;
$$

le complete $\Omega$ considéré contient le voisinage du second ordre des surfaces (5.15) et (5.16).

Par différentiation extérieure des relations (5.15) et (5.16) on obtient

$$
\mathrm{d} c_{1}+2 c_{1}\left(\omega_{11}+\omega_{44}\right)=\bar{\gamma}_{1} \omega_{1}, \quad \mathrm{~d} c_{2}+2 c_{2}\left(\omega_{11}+\omega_{44}\right)=\bar{\gamma}_{2} \omega_{1} .
$$

Les coordonnées locales de ce complexe-là sont déterminées par la relation (2.23) et l'on a $\left(\alpha_{1} \alpha_{2}-\beta_{1} \beta_{2} \neq 0, \sigma \neq 0\right.$ arb.)

où

$$
\begin{aligned}
\Omega= & \sigma\left\{\left(\beta_{1} \beta_{2}-\alpha_{1} \alpha_{2}\right)\left(c_{1}+c_{2}\right)\left[A_{1} A_{2}\right]-2\left(\alpha_{2}+\beta_{1} c_{1} c_{2}\right)\left[A_{1} A_{3}\right]+\right. \\
& \left.+2\left(\beta_{2}+\alpha_{1} c_{1} c_{2}\right)\left[A_{2} A_{4}\right]\right\} \\
\mathrm{d} \Omega= & \sigma\left\{f_{1}\left[A_{1} A_{2}\right]+f_{2}\left[A_{1} A_{3}\right]+f_{3}\left[A_{2} A_{4}\right]+f_{4}\left[A_{1} A_{4}\right]+f_{5}\left[A_{2} A_{3}\right]\right\}+ \\
& +\left(\mathrm{d} \sigma / \sigma-\omega_{11}+\omega_{22}+2 \omega_{33}\right) \Omega \\
\mathrm{d}^{2} \Omega= & \text { comb. lin. } \operatorname{de}\left(\left[A_{1} A_{2}\right],\left[A_{1} A_{3}\right],\left[A_{2} A_{4}\right]\right)+\left[\mathrm{d}\left(\sigma f_{4}\right)+\right. \\
& \left.+\sigma f_{4}\left(\omega_{11}+\omega_{44}\right)+\sigma f_{1} \omega_{2}+\sigma\left(f_{2} \beta_{2}+f_{3} \alpha_{2}\right) \omega_{1}\right]\left[A_{1} A_{4}\right]+ \\
& +\left[\mathrm{d}\left(\sigma f_{5}\right)+\sigma f_{5}\left(\omega_{22}+\omega_{33}\right)-\sigma f_{1} \omega_{1}+\sigma\left(f_{2} \alpha_{1}+f_{3} \beta_{1}\right) \omega_{2}\right] \\
& \cdot\left[A_{2} A_{3}\right]+2 \sigma\left(\alpha_{1} \alpha_{2}-\beta_{1} \beta_{2}\right)\left(c_{1} \omega_{1}-\omega_{2}\right)\left(c_{2} \omega_{1}-\omega_{2}\right)\left[A_{3} A_{4}\right],
\end{aligned}
$$

$$
\begin{aligned}
f_{1}= & {\left[\left(\bar{\gamma}_{1}+\bar{\gamma}_{2}\right)\left(\beta_{1} \beta_{2}-\alpha_{1} \alpha_{2}\right)-2\left(\alpha_{2}+\beta_{1} c_{1} c_{2}\right) \gamma_{1}-\alpha_{2}\left(c_{1}+c_{2}\right) \gamma_{2}-\right.} \\
& -2\left(\beta_{2}+\alpha_{1} c_{1} c_{2}\right) \gamma_{2}+\left(c_{1}+c_{2}\right) \beta_{2} \gamma_{4}-\alpha_{2}\left(c_{1}+c_{2}\right) \gamma_{6}+ \\
& \left.+\beta_{1}\left(c_{1}+c_{2}\right) \gamma_{8}\right] \omega_{1}+\left[-\beta_{1}\left(c_{1}+c_{2}\right) \gamma_{1}-2\left(\alpha_{2}+\beta_{1} c_{1} c_{2}\right) \gamma_{2}-\right. \\
& -\alpha_{1}\left(c_{1}+c_{2}\right) \gamma_{3}-2\left(\beta_{2}+\alpha_{1} c_{1} c_{2}\right) \gamma_{4}-\alpha_{2}\left(c_{1}+c_{2}\right) \gamma_{5}+ \\
& \left.+\beta_{2}\left(c_{1}+c_{2}\right) \gamma_{7}\right] \omega_{2}, \\
f_{2}= & -2\left[\beta_{1}\left(\bar{\gamma}_{1} c_{2}+\bar{\gamma}_{2} c_{1}\right)-\gamma_{4} c_{1} c_{2}+\gamma_{6}\right] \omega_{1}-2\left[\gamma_{3}+c_{1} c_{2} \gamma_{7}\right] \omega_{2}, \\
f_{3}= & 2\left[\alpha_{1}\left(\bar{\gamma}_{1} c_{2}+\bar{\gamma}_{2} c_{1}\right)+\gamma_{2} c_{1} c_{2}+\gamma_{8}\right] \omega_{1}+2\left[-\gamma_{1}+c_{1} c_{2} \gamma_{5}\right] \omega_{2}, \\
f_{4}= & \left(\beta_{1} \beta_{2}-\alpha_{1} \alpha_{2}\right)\left[\left(c_{1}+c_{2}\right) \omega_{2}-2 c_{1} c_{2} \omega_{1}\right], \\
f_{5}= & -\left(\beta_{1} \beta_{2}-\alpha_{1} \alpha_{2}\right)\left[\left(c_{1}+c_{2}\right) \omega_{1}-2 \omega_{2}\right] .
\end{aligned}
$$

Le point (5.18) décrit dans l'espace de Klein la surface $\Omega\left(c_{1}, c_{2}\right)$ qui ne possède auicun réseau conjugué $\left(\alpha_{1} \alpha_{2}-\beta_{1} \beta_{2} \neq 0\right)$; les points de la surface $\Omega\left(c_{1}, c_{2}\right)$ et les droites de la congruence $L$ sont dans une correspondence biunivoque. 
L'équation différentielle

$$
\omega_{2}=c_{3} \omega_{1}, \quad c_{3} \neq 0
$$

détermine dans l'espace de Klein d'une part des décompositions des congruences $\Omega\left(c_{1}\right)$ et $\Omega\left(c_{2}\right)$ en surfaces réglées et d'autre part une courbe sur la surface $\Omega\left(c_{1}, c_{2}\right)$ que nous voulons désigner par $\Omega\left(c_{1}, c_{2} ; c_{3}\right)$. Les points de cette courbe sont des images secondaires des complexes tangents qui possèdent le voisinage du $2^{\mathrm{e}}$ ordre des surfaces (5.15) et (5.16) le long des droites de la surface (5.21) de la congruence $L$. L'espace qui joint le plan osculateur (donné par les points $(5.18)-(5.20)$, où on a substitué d'après (5.21)) et l'espace tangent à quatre dimensions (5.2) (c.-à-d. l'enveloppe linéaire) est en général l'espace $\bar{P}_{5}$. La condition nécessaire et suffisante pour que cette enveloppe soit de dimension plus petite, c.-à-d. pour que le plan mentionné soit situé dans l'espace (5.2) est que l'on ait $c_{3}=c_{1}$ ou $c_{3}=c_{2}$. On peut résumer les résultats précédents de la manière suivante:

Les courbes $\Omega\left(c_{1}, c_{2} ; c_{1}\right)$ et $\Omega\left(c_{1}, c_{2} ; c_{2}\right)$ situées sur la surface $\Omega\left(c_{1}, c_{2}\right)$ sont les courbes quasiasymptotiques $\left.\gamma_{1,2}{ }^{9}\right)$ de la variété $(\Omega)$.

Comme une conséquence de ce théorème on a:

Les surfaces réglées (5.15) et (5.16) des congruences $\Omega\left(c_{1}\right)$ et $\Omega\left(c_{2}\right)$ coupent la surface $\Omega\left(c_{1}, c_{2}\right)$ - qui est leur intersection - aux courbes quasiasymptotiques $\gamma_{1,2}$ de la variété $(\Omega)$.

On peut aussi énoncer le théorème précédent de la manière suivante:

La condition nécessaire et suffisante pour que les images secondaires des complexes tangent $\Omega\left(c_{1}, c_{2}\right)$ qui possèdent le voisinage du second ordre des surfaces réglées (5.15) et (5.16) de la congruence L décrivent sur la variété $(\Omega)$ les courbes quasiasymptotiques $\gamma_{1,2}$ est: le complexe $\Omega\left(c_{1}, c_{2}\right)$ se déplace le long de la surface réglée de la couche (5.15) ou (5.16). L'ensemble des courbes quasiasymptotiques dépend de deux fonctions d'une variable.

Remarquons encore que dans les relations $(5.15)-(5.21)$ on peut avoir $c_{1}=c_{2}=c$ et alors $\bar{\gamma}_{1}=\bar{\gamma}_{2}=\gamma$; ensuite les courbes quasiasymptotiques $\gamma_{1,2}$ sont situées sur la variété singulière $(F)$.

La variété $(\Omega)$ correspondant aux congruences du type II, resp. V possède les propriétés analogues à celles des variétés correspondant aux congruences du type I $\left(\alpha_{1} \alpha_{2}-\beta_{1} \beta_{2} \neq 0\right)$ et sa variété singulière est tangente à la $K$-quadrique aux $K$-points de la surface décrite par le $K$-point $\left[A_{1} A_{3}\right]$ ou $\left[A_{2} A_{4}\right]$, resp. aux points des deux sur-

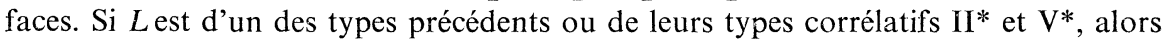

\footnotetext{
$\left.{ }^{9}\right)$ La variété $V_{m}$ est une variété quasiasymptotique $\sigma_{r, s}$ sur la variété $V_{k}\left(\supset V_{m}\right), m<k$, si la dimension de l'espace linéaire (de l'enveloppe linéaire) déterminé par l'espace osculateur $S(r)$ d'ordre $r$ de la variété $V_{k}$ et par l'espace osculateur $S(s)$ d'ordre $s$ de la variété $V_{m}$ est plus petite que la dimension maximum correspondant aux valeurs $k, m, r, s$. Si $m=1$ on obtient les courbes quasiasymptotiques $\gamma_{r, s}$.
} 
les plans infiniment voisins de la congruence $\Lambda$ possèdent juste un point commun parce que le rang de la matrice (5.5) (après l'anulation des invariants correspondants $\alpha_{i}$ ou $\beta_{i}$ ) est 5 .

Dans le cas des congruences $W$, les plans de la congruence $\Lambda$ enveloppent une surface qui possède un réseau conjugué et la surface singulière $(F)$ dégénère en deux congruences de droites qui sont tangentes aux courbes de ce réseau.

Si $L$ est une congruence d'un autre type, alors la variété $(\Omega)$ et la variété singulière $(F)$ dégénèrent de plus en plus comme on voit sans peine en vertu des résultats du chap. 4.

\section{Littérature}

[1] E. Čech: Transformations développables des congruences de droites. Чехосл. мат. журнал, $6(81), 1956,260-286$.

[2] С. П. Фиников: Теория конгруэнций. Москва, 1950.

[3] V. Horák: Les complexes osculateurs des congruences de droites. Чехосл. мат. журнал, $11(86), 1961,440-460$.

[4] V. Horák: Contribution à la théorie des déformations projectives des congruences $W$. Чехосл. мат. журнал, 12 (87), 1962, 251-273.

\section{Резюме}

\section{ЛИНЕЙНЫЕ КАСАТЕЛЬНЫЕ КОМПЛЕКСЫ ПРЯМОЛИНЕЙНЫХ КОНГРУЭНЦИЙ}

\section{ВЛАДИМИР ГОРАК (Vladimír Horák), Брно}

Автором исследуются многообразия вторичных образов Клейна касательных комплексов непараболических прямолинейных конгруэнций пространства $P_{3}$. Согласно Эд. Чеху мы различаем 10 типов непараболических прямолинейных конгруэнций, геометрическое описание которых приводится в п. 1 этой работы.

Пусть $\left[A_{1} A_{2}\right]$ - луч какой-либо конгруэнции $L$ и $\left[A_{1} A_{3}\right],\left[A_{2} A_{4}\right]$ - лапласовские преобразования этого луча (если фокальная поверхность является развертывающейся поверхностью или кривой (прямой), то прямая $\left[A_{1} A_{3}\right]$ или $\left[A_{2} A_{4}\right]$ представляет собой образующую прямую этой развертывающейся поверхности или касательной к указанной кривой в соответствующем фокусе).

Каждому лучу $\left[A_{1} A_{2}\right]$ конгруэнции $L$ принадлежит $\infty^{2}$ линейных комплек$\operatorname{coв} \Omega$, содержащих окрестность первого порядка этого луча (т. наз. касательных комплексов); вторичные образы Клейна зтих комплексов заполняют плоскость ( $\left.\left[A_{1} A_{2}\right],\left[A_{1} A_{3}\right],\left[A_{2} A_{4}\right]\right)$, являющуюся касательной плоскостью гиперквадрииКлейна (K-квадрики) в $K$-точке $\left[A_{1} A_{2}\right]$; эта плоскость сопряжена с касательной плоскостью $K$-поверхности, которая является прямым клейновским 
образом конгруэнции $L$ на $K$-квадрике. Если $L$ не является конгруэнцией $W$ (тип I), или конгруэнцией типа IV, или не принадлежит линейному комплексу, то ни один из касательных комплексов не содержит окрестности второго порядка прямой $\left[A_{1} A_{2}\right]$, но содержит только окрестность второго порядка двух линейчатых поверхностей (которые могут и совпадать) конгруэнции $L$.

Вторичные образы касательных комплексов, содержащих окрестность второго порядка одной и только одной линейчатой поверхности (точнее двух совпадающих поверхностей) конгруэнции $L$, заполняют в плоскости ([ $\left.A_{1} A_{2}\right]$, $\left.\left[A_{1} A_{3}\right],\left[A_{2} A_{4}\right]\right)$ коническое сечение $F$, для которого в общем случае точка $\left[A_{1} A_{2}\right]$ и прямая $\left(\left[A_{1} A_{3}\right],\left[A_{2} A_{4}\right]\right)$ являются полюсом и полярой.

Каждому из упомянутых десяти типов конгруэнций (причем у конгруэнций типа I нужно различать конгруэнции общего вида и конгруэнции $W$ и $V$ ), соответствует или регулярное или особое коническое сечение $F$, Занимающее характеристическое инвариантное положение по отношению к основному треугольнику $\left[A_{1} A_{2}\right],\left[A_{1} A_{3}\right],\left[A_{2} A_{4}\right]$. Конгруэнция типа I является конгруэнцией $W$, если и только если $F$ - особое коническое сечение. В общих чертах можно сказать, что коническое сечение $F$ регулярно для конгруэнций типа I, не являющихся $W$, и далее для типов II, II*, V, V*.

Пусть $L-$ конгруэнция типа I, но не является конгруэнцией $W$. Любые две различные точки плоскости ([ $\left.\left.A_{1} A_{2}\right],\left[A_{1} A_{3}\right],\left[A_{2} A_{4}\right]\right)$ являются образами касательных комплексов, которые содержат окрестности второго порядка двух различных пар линейчатых поверхностей конгруэнции $L$, проходящих через прямую $\left[A_{1} A_{2}\right]$. Вторичные образы касательных комплексов, содержащих окрестности второго порядка какой-либо фиксированной линейчатой поверхности конгруэнции $L$, заполняют в указанной плоскости касательную к коническому сечению $F$. Если $L-$ конгруэнция $W$, то образы всех касательных комплексов, содержащих окрестность второго порядка какой-либо линейчатой поверхности, лежат на прямой, проходящей через особую точку конического сечения $F$; эти $\infty^{1}$ комплексов содержат одновременно окрестность второго порядка дальнейшей однозначно определенной поверхности конгруэнции $L$. Итак, структуры окрестности первого порядка конгруэнций $W$ и остальных конгруэнций типа I различны. Автор истолковывает известный инвариант Вельша конгруэнций типа I, как сложное отношение вторичных образов некоторых геометрически замечательных комплексов, и определяет другие геометрически замечательные комплексы, вторичные образы которых обладают инвариантным сложным отношением, являющимся, следовательно, функцией инварианта Вельша.

Вторичные образы касательных комплексов конгруэнций типа I (за исключением $W$ ) заполняют в пространстве Клейна $\bar{P}_{5}$ четырехмерное точечное многообразие $(\Omega)$, являющееся одновременно конгруэниией плоскостей $\Lambda$; каждая плоскость конгруэнции $\Lambda$ представляет собой множество вторичных образов касательных комплексов к одной и той же прямой, так что лучи кон- 
груэнции $L$ и плоскости конгруэнции $\Lambda$ связаны взаимно однозначным соответствием. Точки конического сечения $F$ в плоскости ([ $\left.\left.A_{1} A_{2}\right],\left[A_{1} A_{3}\right],\left[A_{2} A_{4}\right]\right)$ являются фокусами этой плоскости конгруэнции $A ; \infty^{2}$ конических сечений $F$ заполняет трехмерное особое многообразие $(F)$ многообразия $(\Omega)$. Любые две совпадающие плоскости конгруэнции $\Lambda$ имеют, вообще, ровно одну общую точку; для того, чтобы эти плоскости пересекались в прямой, необходимо и достаточно, чтобы $L$ была конгруэнцией $W$.

Далее автор исследует развертывающиеся линейчатые поверхности многообразия $(\Omega)$, показывает, что в пространстве $P_{3}$ им соответствуют т. наз. конгруэнции Сегре, линейчатые фокальные поверхности которых касаются вдоль некоторых кривых фокальных поверхностей конгруэнции $L$ и, наконец, определяет квазиасимптотические кривые $\gamma_{1,2}$ многообразия $(\Omega)$ и описывает их геометрическое значение для конгруэнции $L$. 